\title{
Eigenfunction Expansions Associated with Second-order Differential Equations for Hilbert Space-valued Functions*
}

\author{
By \\ Yoshimi SaItō
}

\section{§o. Introduction}

In this paper we shall consider differential operators of the form

$$
\mathscr{L}=-\frac{d^{2}}{d r^{2}}+A(r) \quad 0 \leqq r<\infty,
$$

where for each $r \in[0, \infty) A(r)$ is an operator in a Hilbert space $H$ and $\mathscr{L}$ acts on $H$-valued functions on $[0, \infty)$. Restricting the domain of $\mathscr{L}$ appropriately, we can regard $\mathscr{L}$ as an operator in $\mathfrak{h}=L_{2}(0, \infty ; H)$. Our purpose is to develop an eigenfunction expansion theory associated with the differential operator $\mathscr{L}$.

If $\operatorname{dim} H=1$, i.e. $H \cong \mathbf{C}$, then $\mathscr{L}$ is an ordinary second-order differential operator and $A(r)$ is simply an operator of multiplication by a function $q(r)$. For real-valued $q(r)$ a rather complete eigenfunction expansion theory has been worked out by Weyl [10], Stone [8], Titchmarsh [9], Kodaira [4], [5] and others. But when $H$ is an infinite-dimensional Hilbert space, it seems that no complete theory, comparable with the one for ordinary differential operators, has been presented.

Rofe-Beketov [7] considers the case where $A(r)$ is a bounded selfadjoint operator-valued function on $[0, \infty)$ which is continuous in the uniform operator topology. He shows that there exist a non-negative definite, bounded operator-valued, interval function $\rho(I), I \subset \mathbf{R}$, and a bounded operator-valued function $\omega(r, \lambda)$ on $[0, \infty)$, satisfying

Received October 29, 1970.

* The research was supported in part by the Sakkōkai Foundation. 


$$
\left\{\begin{array}{l}
(\mathscr{L}-\lambda) \omega(r, \lambda)=0, \\
\omega^{\prime}(0, \lambda)-C \omega(0, \lambda)=0,
\end{array}\right.
$$

where $C$ is a bounded, self-adjoint operator on $H . \quad \omega(r, \lambda)$ and $\rho(I)$ are incorporated to give an eigenfunction expansion of an arbitrary function $F \in L_{2}(0, \infty ; H)=\mathfrak{h}$ in the following sense: Let $\mathfrak{h}_{\rho}=L_{2}(-\infty, \infty ; H$; $\rho(d \mu))$ and $\mathscr{F}_{\mu}(F)=\int_{0}^{\infty} \omega^{*}(r, \mu) F(r) d r$, where $\omega^{*}(r, \mu)$ denotes the adjoint of $\omega(r, \lambda)$. Then the mapping $F \rightarrow \mathscr{F}_{\mu}(F)$ is isometric from $\mathfrak{h}$ into $\mathfrak{h}_{\rho}$. He also discusses the inverse problem in which one determines $A(r)$ and $C$ from $\rho(I)$, and the case of $A(r)$ being not necessarily self-adjoint.

V. Gorbačuk and M. Gorbačuk [2] obtain similar results to RofeBeketov $[7]$ for the operator $\mathscr{L}$ in which $A(r)$ is replaced by $-A+Q(r)$, where $A$ is a lower semi-bounded, self-adjoint operator in $H$ and $Q(r)$ is a bounded, self-adjoint operator on $H$ for each $r \in[0, \infty)$. This situation, one should note, makes $\mathscr{L}$ hyperbolic rather than elliptic.

In contrast to Gorbačuk and Gorbačuk's treatment Jäger [3] deals with the elliptic case, i.e. he assumes $A(r)=A_{1}(r)+A_{2}(r)$, where $A_{1}(r)$ is a non-negative, self-adjoint operator in $H$ with domain $\mathscr{D}\left(A_{1}(r)\right)=D$ constant in $r$ and $A_{2}(r)$ is a symmetric operator with domain $\mathscr{D}\left(A_{2}(r)\right)$ $=D$. Under the above assumptions together with some asymptotic and regularity conditions on $A_{1}(r)$ and $A_{2}(r)$ he determines a unique selfadjoint operator which is a restriction of $\mathscr{L}$, and obtains an eigenfunction expansion similar to Rofe-Beketov [7]. The eigenfunctions are obtainable from the Green functions for $\mathscr{L}$ by a limiting process.

Jäger's results are applicable to the Schrödinger operator $-\Delta+q(x)$ in $\mathbf{R}^{3}$ with $q(x)$ bounded by a constant multiple of $(1+|x|)^{-3 / 2-\varepsilon}(\varepsilon>0)$. In this case $\mathfrak{h}=L_{2}(0, \infty ; H)=L_{2}\left(0, \infty ; L_{2}\left(S^{2}\right)\right)$, where $S^{2}$ is the 2 sphere, and $A_{1}(r)$ and $A_{2}(r)$ are taken to be $\frac{A_{0}}{r^{2}}$ and $q(r \omega), \omega \in S^{2}, A_{0}$ being the negative of the Laplace-Beltrami operator on $S^{2}$.

In the present paper we shall consider the eigenfunction expansion for any self-adjoint restriction $L_{0}$ in $\mathfrak{h}$ of $\mathscr{L}$ with bounded $A(r)$ by a method somewhat different from those explained above. 
In $\S 1$ we introduce the "eigenoperator" $\phi(r, \lambda)$ of $\mathscr{L}$ which satisfies the boundary condition at $r=0$

$$
B F^{\prime}(0)-C F(0)=0
$$

where $B$ and $C$ are bounded, self-adjoint operators on $H . \phi(r, \lambda)$ is a bounded operator on $H$ for each pair $(r, \lambda) \in[0, \infty) \times \mathbf{C}$ and satisfies

$$
\left\{\begin{array}{l}
(\mathscr{L}-\lambda) \phi(r, \lambda)=0, \\
B \phi^{\prime}(0, \lambda)-C \phi(0, \lambda)=0 .
\end{array}\right.
$$

$\S 2$ prepares Green's formula for $H$-valued functions which will be used mainly in $§ 3$.

In $\S 3$ we define an operator $L$ in $\mathfrak{h}$ which is a restriction of $\mathscr{L}$ and investigate the properties of $L$. Roughly speaking, the domain of $L$ is the set of all $H$-valued functions $F(r)$ on $[0, \infty)$ such that $F(r)$ satisfies the boundary condition (0.3) and $F$ and $\mathscr{L} F$ belong to $\mathfrak{h}$.

Now let $L_{0}$ be a self-adjoint restriction of $L$, if any. The eigenfunction expansion problem associated with $L_{0}$ is discussed in $\S 4$. Denoting by $E(\mu)$ the resolution of the identity corresponding to $L_{0}$, we can show that for any half-open interval $I=\left(\mu_{1}, \mu_{2}\right]$ there exists a bounded operator $\xi(I)$ taking $\mathfrak{h}$ into $H$ such that

$$
E(I) F(r)=\int_{I} \phi(r, \mu) \xi(d \mu) F
$$

holds for any $F \in \mathfrak{h}$. The relation $(0.5)$ is a generalization of Kodaira's formula $([4]$, p. $188,(4.12)$ or $[5], \quad$ p. $930,(2.6))$. A non-negative definite, bounded operator-valued, interval function $\rho(I)$ is introduced by

$$
\rho(I)=\xi(I) \xi^{*}(I),
$$

where $\xi^{*}(I)$ is the adjoint of $\xi(I)$ taking $H$ into $\mathfrak{h}$. We denote $L_{2}(-\infty$, $\infty ; H ; \quad \rho(d \mu))$ by $\mathfrak{h}_{\rho}$. The eigenfunction expansion for $L_{0}$ takes the following form (Theorem 4.18): Define the "generalized Fourier transform" $\mathscr{F}$ associated with $L_{0}$ by

$$
(\mathscr{F} F)(\mu)=\int_{0}^{\infty} \phi^{*}(r, \mu) F(r) d r \quad \text { in } \mathfrak{h}_{\rho} .
$$


Then $\mathscr{F}$ is a unitary operator from $\mathfrak{h}$ onto $\mathfrak{h}_{p}$ and we have

$$
\left\{\begin{array}{c}
E(I)=\mathscr{F}^{*} \chi_{I} \cdot \mathscr{F}, \\
L_{0}=\mathscr{F}^{*} \mu \cdot \mathscr{F},
\end{array}\right.
$$

where $\chi_{I}$ is the characteristic function of the interval $I$.

In the Appendix we study Riemann-Stieltjes integrals involving vectorvalued functions and operator-valued measuses, and we give the proofs of some lemmas which are stated in $§ 4$.

The author wishes to express his sincere thanks to Professor $\mathrm{H}$. Yoshizawa for his unceasing encouragement and to Professor T. Ikebe for his kind advices and discussions.

\section{§1. The Equation $\left(-\frac{d^{2}}{d r^{2}}+A(r)-\lambda\right) F(r)=0$}

Let $H$ be a separable Hilbert space with the norm \|\|$_{H}$ and the inner product $(,)_{H}$. For $H$-valued functions $F(r)$ and $G(r)$ on $(0, \infty)$ introduce the norm and inner product by

$$
\|F\|_{\mathfrak{h}}=\left[\int_{0}^{\infty}\|F(r)\|_{H}^{2} d r\right]^{\frac{1}{2}},(F, G)_{\mathfrak{h}}=\int_{0}^{\infty}(F(r), G(r))_{H} d r .
$$

$\mathfrak{h}$ will denote the Hilbert space of all (equivalence classes of) $H$-valued functions $F(r)$ on $(0, \infty)$ with $\|F\|_{\mathfrak{h}}<\infty$, i.e. $\mathfrak{h}=L_{2}(0, \infty ; H)$. The subscripts $H$ and $\mathfrak{h}$ will be omitted where no fear of confusion occurs.

Let $\mathbf{B}$ be the set of all everywhere defined, bounded, linear operators on $H$, and let $A(r)$ be a $\mathbf{B}$-valued function on $[0, \infty)$. For $A(r)$ we make the following

Assumption 1.1. (1) For each $r \in[0, \infty) A(r)$ is a bounded, self. adjoint operator on $H$.

(2) For arbitrary $r \in[0, \infty)$ we have $\int_{0}^{r}\|A(t)\| d t<\infty$, that is, $\|A(r)\|$ is locally integrable on $[0, \infty)$, where $\|A(r)\|$ denotes the operator norm of $A(r)$.

(3) For any $r \in[0, \infty)$ there exists a positive number $C_{r}$ such that 


$$
\int_{0}^{r}(A(s) F(s), F(s))_{H} d s \geqq-C_{r} \int_{0}^{r}\|F(s) !\|_{H}^{2} d s
$$

for all $F \in \mathfrak{G}$ such that $\|F(r)\|_{H}$ is a bounded function on $[0, \infty){ }^{1)}$

Now let us consider the initial value problem for the equation

$$
\left(-\frac{d^{2}}{d r^{2}}+A(r)-\lambda\right) F(r)=0
$$

Definition 1.2. Denote by $\mathbf{D}$ the set of all H-valued functions $F(r)$ on $[0, \infty)$ satisfying the following (1) and (2):

(1) $F(r)$ is strongly continuously differentiable on $[0, \infty)$ with its strong derivative $F^{\prime}(r)$.

(2) $F^{\prime}(r)$ is weakly absolutely continuous on every finite interval in $[0, \infty)$, and $F^{\prime}(r)$ is weakly differentiable almost everywhere on $[0, \infty)$ with its weak derivative $F^{\prime \prime}(r)^{2)}$ such that $\left\|F^{\prime \prime}(r)\right\|_{H}$ is locally integrable on $[0, \infty)$. Then let us define a differential operator $\mathscr{L}$ by

$$
\left\{\begin{array}{l}
\mathscr{D}(\mathscr{L})=\mathbf{D}^{3)} \\
\mathscr{L} F(r)=-F^{\prime \prime}(r)+A(r) F(r) .
\end{array}\right.
$$

Let $u_{0}, v_{0} \in H$ and let $\lambda$ be a complex number. Then an H-valued function $F(r)$ on $[0, \infty)$ is called a solution of (1.3) with (initial) data $\left\{u_{0}, v_{0}\right\}$ if $F \in \mathbf{D}, F(0)=u_{0}$ and $F^{\prime}(0)=v_{0}$, and the equation

$$
(\mathscr{L}-\lambda) F(r)=0
$$

holds for alinost all $r \in[0, \infty)$.

Concerning Definition 1.2, we have the following

1) In $\S 1$ and $\S 2$ we shall not use (3) of Assumption 1.1.

2) Here and henceforth we say that an H-valued function $G(r)$ on an interval $I$ is weakly absolutely continuous almost everywhere on I with its weak derivative $G^{\prime}(r)$ if and only if there exists a null set $e$ in $I$ such that

$$
\frac{d}{d r}(G(r), u)_{\mathrm{H}}=\left(G^{\prime}(r), u\right)_{\mathrm{H}}
$$

holds for any $r \in I-e$ and any $u \in H$.

3) We denote by $\mathscr{D}(T)$ the domain of $T$. 
Lemma 1.3. Let $F(r) \in \mathbf{D}$ and let $F^{\prime}(r)$ be as in Definition 1. 2. Then $F^{\prime}(r)$ is strongly absolutely continuous on every finite interval in $[0, \infty)$.

Lemma 1.4. Suppose that an H-valued function $G(r)$ is weakly absolutely continuous on every finite interval in $[0, \infty)$. Moreover suppose that there exists an $H$-valued function $G_{1}(r)$ on $[0, \infty)$ such that $\left\|G_{1}(r)\right\|_{H}$ is locally integrable on $[0, \infty)$ and for any $u \in H$ we have

$$
\frac{d}{d r}(G(r), u)_{H}=\left(G_{1}(r), u\right)_{H}
$$

for almost all $r \in[0, \infty)$, where the exceptional set may depend on $u$. Then $G(r)$ is weakly differentiable almost everywhere on $[0, \infty)$ with its derivative $G^{\prime}(r)$ and we have $G^{\prime}(r)=G_{1}(r)$ for almost all $r \in[0, \infty)$.

Remark 1.5. It follows from Lemma 1.4 that we can replace the condition (2) in Definition 1.2 by the following, apparently weaker, condition:

$(2)^{\prime} F^{\prime}(r)$ is weakly absolutely continuous on every finite interval in $[0, \infty)$, and there exists an $H$-valued function $F^{\prime \prime}(r)$ on $[0, \infty)$ such that $\left\|F^{\prime \prime}(r)\right\|_{H}$ is locally integrable on $[0, \infty)$ and for any $u \in H$ we have $\frac{d}{d r}$ $\left(F^{\prime}(r), u\right)_{H}=\left(F^{\prime \prime}(r), u\right)_{H}$ for almost all $r \in[0, \infty)$, where the exceptional set may depend on $u$.

Proof of Lemma 1.3. It follows from Definition 1.2, (2) that for all $u \in H$ and $0 \leqq r_{1}<r_{2}$

$$
\left(F^{\prime}\left(r_{2}\right)-F^{\prime}\left(r_{1}\right), u\right)_{H}=\int_{r_{1}}^{r_{2}}\left(F^{\prime \prime}(t), u\right)_{H} d t
$$

where $F^{\prime \prime}(r)$ is the weak derivative of $F^{\prime}(r)$. Hence we have

$$
\left|\left(F^{\prime}\left(r_{2}\right)-F^{\prime}\left(r_{1}\right), u\right)_{H}\right| \leqq\|u\|_{H} \int_{r_{1}}^{r_{2}}\left\|F^{\prime \prime}(t)\right\|_{H} d t
$$

Since $u \in H$ is arbitrary, we obtain from (1.8) 


$$
\left\|F^{\prime}\left(r_{2}\right)-F^{\prime}\left(r_{1}\right)\right\|_{H} \leqq \int_{r_{1}}^{r_{2}}\left\|F^{\prime \prime}(t)\right\|_{H} d t
$$

which completes the proof.

Q.E. D.

Proof of Lemma 1.4. Let $\left\{u_{n}\right\}_{u=1}^{\infty}$ be a complete, orthonormal basis of $H$. Then for each $n$ there exists a null set $e_{n}$ in $[0, \infty)$ such that

$$
\frac{d}{d r}\left(G(r), u_{n}\right)_{H}=\left(G_{1}(r), u_{n}\right)_{H}
$$

holds for $r \in[0, \infty)-e_{n}$. On the other hand it follows from the local integrability of $\left\|G_{1}(r)\right\|_{H}$ that there exists a null set $e_{0}$ such that

$$
\frac{d}{d r} \int^{r}\left\|G_{1}(t)\right\|_{H} d t=\left\|G_{1}(r)\right\|_{H}
$$

holds for $r \in[0, \infty)-e_{0}$. Put $e=\bigcup_{m=0}^{\infty} e_{m}$ and let $r \in[0, \infty)-e$. Then for $u \in H$ we have

$$
\begin{aligned}
\left(\frac{1}{h}\{G(r+h)-G(r)\}-G_{1}(r), u\right)_{H} \\
=\left(\frac{1}{h}\{G(r+h)-G(r)\}-G_{1}(r), u^{(N)}\right)_{H} \\
+\left(\frac{1}{h}(G(r+h)-G(r)), \tilde{u}^{(N)}\right)_{H} \\
+\left(-G_{1}(r), \tilde{u}^{(N)}\right)_{H} \equiv I_{1}+I_{2}+I_{3},
\end{aligned}
$$

where $N$ is an arbitrary positive integer and

$$
u^{(N)}=\sum_{n=1}^{N}\left(u, u_{n}\right)_{H} u_{n}, \tilde{u}^{(N)}=u-u^{(N)} .
$$

Since $r \notin e_{n}(n=1,2, \ldots)$, we have

$$
\left|I_{1}\right| \rightarrow 0(h \rightarrow 0)
$$

for any fixed $N$.

$I_{2}$ and $I_{3}$ are estimated as follows: 


$$
\left\{\begin{aligned}
\left|I_{2}\right|=\left|\frac{1}{h} \int_{r}^{r+h}\left(G_{1}(t), \tilde{u}^{(N)}\right)_{H} d t\right| \\
\leqq\left\|\tilde{u}^{(N)}\right\|_{H} \frac{1}{|h|} \int_{r-|h|}^{r+|h|}\left\|G_{1}(t)\right\|_{H} d t \rightarrow 0 \quad(N \rightarrow \infty) \\
\left|I_{3}\right| \leqq\left\|\tilde{u}^{(N)}\right\|_{H}\left\|G_{1}(r)\right\|_{H \rightarrow 0} \quad(N \rightarrow \infty)
\end{aligned}\right.
$$

Noting $r \notin e_{0}$, we can see that $\frac{1}{|h|} \int_{r-|h|}^{r+|h|}\left\|G_{1}(t)\right\|_{H} d t$ is bounded when $h \rightarrow 0$. Therefore it follows from (1.14) and (1.15) that for any $u \in H$ and $r \notin e$

$$
\frac{d}{d r}(G(r), u)_{H}=\left(G_{1}(r), u\right)_{H}
$$

holds.

Q.E.D.

We shall show the existence of $\mathbf{B}$-valued functions $\omega(r, \lambda)$ on $[0, \infty)$ $\times \mathbf{C}^{4)}$ such that for any $u \in H \omega(r, \lambda) u$ is the solution of (1.3) with suitable initial data. Then we shall investigate the properties of $\omega(r, \lambda)$.

Proposition 1.6. Let $S, T \in \mathbf{B}$. Then there exists a B-valued function $\omega(r, \lambda)=\omega(r, \lambda ; S, T)$ on $[0, \infty) \times \mathbf{C}$ satisfying the following (1) (5):

(1) For any $u \in H \omega(r, \lambda) u$ is a unique solution of (1.3) with data $\{S u, T u\}$.

(2) $\omega(r, \lambda)$ is continuous in the uniform topology of $\mathbf{B}$ on $[0, \infty)$ $\times \mathbf{C}$ and $\omega(0, \lambda)=S$.

(3) For each $\lambda \in \mathbf{C} \omega(r, \lambda)$ is continuously differentiable on $[0, \infty)$ in the uniform topology of $\mathbf{B}$ with its derivative $\omega^{\prime}(r, \lambda)$ in $\mathbf{B}$, and $\omega^{\prime}(0, \lambda)=T$.

(4) For each $\lambda \in \mathbf{C} \omega^{\prime}(r, \lambda)$ is weakly absolutely continuous on every finite interval in $[0, \infty)$. There exists a null set $e$ in $[0, \infty)$ such that for any $r \in[0, \infty)-e \omega^{\prime}(r, \lambda)$ is weakly differentiable with its derivative $\omega^{\prime \prime}(r, \lambda) \in \mathbf{B}$. The exceptional set $\boldsymbol{e}$ does not depend on $\lambda \in \mathbf{C}$. $\left\|\omega^{\prime \prime}(r, \lambda)\right\|$ is locally integrable on $[0, \infty)$ for each $\lambda \in \mathbf{C}$ and we have

4) C means the set of all complex numbers. 


$$
-\omega^{\prime \prime}(r, \lambda)+A(r) \omega(r, \lambda)=\lambda \omega(r, \lambda)
$$

for all $r \in[0, \infty)-e$ and $\lambda \in \mathbf{C}$.

(5) $\omega(r, \lambda), \omega^{\prime}(r, \lambda)$ and $\omega^{\prime \prime}(r, \lambda)$ are B-valued, entire funclions for cach $r \in[0, \infty)$.

From Proposition 1.6 we can easily see the following

Corollary 1.7. Let $u_{0}, v_{0} \in H$ : Then there exists a unique solution of (1.3) with data $\left\{u_{0}, v_{0}\right\}$.

Proof of Proposition 1.6. First we shall prove the uniqueness of $\omega(r, \lambda)$. To this end it is sufficient to show that if $F(r)$ is the solution of (1.3) with data $\{0,0\}$, then $F(r) \equiv 0$. Multiplying the equation

$$
-\left(F^{\prime \prime}(t), u\right)_{H}+(A(t) F(t), u)_{H}=(\lambda F(t), u)_{H}
$$

by $\frac{\sin \sqrt{\lambda}(r-t)}{\sqrt{\lambda}}$ and integrating, we obtain

$$
(F(r), u)_{H}=\int_{0}^{r} \frac{\sin \sqrt{\lambda}(r-t)}{\sqrt{\lambda}}(A(t) F(t), u)_{H} d t,
$$

where $u$ is an arbitrary element of $H$. Let us take $r_{0}>0$. Then taking account of (1) of Definition 1.2 we have a positive number $C\left(r_{0}\right)$ such that $\|F(r)\|_{H} \leqq C\left(r_{0}\right)$ is valid for all $r \in\left[0, r_{0}\right]$. Hence we obtain from (1.19)

$$
\begin{aligned}
\left|\left(F^{\prime}(r), u\right)_{H}\right| & \leqq \int_{0}^{r}\left|\frac{\sin \sqrt{\lambda}(r-t)}{\sqrt{\lambda}}\right|\|A(t)\| C\left(r_{0}\right)\|u\| d t \\
& \cong g(r) C\left(r_{0}\right)\|u\|
\end{aligned}
$$

for $r \in\left[0, r_{0}\right]$. Here we put

$$
g(r)=\left\{\begin{array}{ll}
\frac{e^{b r}}{|\sqrt{\lambda}|} \int_{0}^{r}|| A(t) \| d t & \text { if } \lambda \neq 0, \\
r \int_{0}^{r}\|A(t)\| d t & \text { if } \lambda=0,
\end{array} \quad(b=|\operatorname{Im} \sqrt{\lambda}|) .\right.
$$

Putting $u=F(r)$ in (1.20), we have 


$$
\|F(r)\| \leqq g(r) C\left(r_{0}\right) .
$$

Repeating this argument, we have for any positive integer $n$ and $r \in\left[0, r_{0}\right]$.

$$
\begin{cases}\|F(r)\| \leqq \frac{C\left(r_{0}\right)}{n !} e^{b r}\left[\int_{0}^{r} \frac{\|A(t)\|}{|\sqrt{\lambda}|} d t\right]^{n} & \text { if } \lambda \neq 0, \\ \|F(r)\| \leqq \frac{C\left(r_{0}\right)}{n !}\left[r \int_{0}^{r}\|A(t)\| d t\right]^{n} & \text { if } \lambda=0,\end{cases}
$$

and hence $F(r)=0$ on $\left[0, r_{0}\right]$. Since $r_{0}$ is arbitrary, we can conclude $F(r) \equiv 0$ on $[0, \infty)$.

Next let us prove the existence of $\omega(r, \lambda ; S, T)$. If we can show the existence of $\omega(r, \lambda ; I, 0)$ and $\omega(r, \lambda ; 0, I)$ satisfying $(1) \sim(5)$ in Proposition 1.6, then we obtain from the uniqueness of $\omega(r, \lambda ; S, T)$ and the linearity of the equation (1.3)

$$
\omega(r, \lambda ; S, T)=\omega(r, \lambda ; I, 0) S+\omega(r, \lambda ; 0, I) T,
$$

and it is easy to see that $\omega(r, \lambda ; S, T)$ also satisfies (1) (5). Accordingly we shall show the existence of $\omega(r, \lambda ; I, 0)$. The existence of $\omega(r, \lambda ; 0, I)$ is proved in a similar way. The proof is divided into the following four steps.

(I) Let $u \in H$ and $\lambda \in \mathbf{C}$. We consider the integral equation

$$
F(r)=\cos \sqrt{\lambda} r \cdot u+\int_{0}^{r} \frac{\sin \sqrt{\lambda}(r-t)}{\sqrt{\lambda}} A(t) F(t) d t
$$

in the strong sense. We can solve (1.25) by successive approximation. Denoting the unique solution of (1.25) by $F(r)=F(r, \lambda, u)$, we have

$$
F(r)=F(r, \lambda, u)=\sum_{n=0}^{\infty} F_{n}(r, \lambda, u) \quad \text { in } H,
$$

where

$$
\left\{\begin{array}{l}
F_{0}(r, \lambda, u)=\cos \sqrt{\lambda} r \cdot u \\
F_{n}(r, \lambda, u)=\int_{0}^{r} \frac{\sin \sqrt{\lambda}(r-t)}{\sqrt{\lambda}} A(t) F_{n-1}(t, \lambda, u) d t \quad(n=1,2, \ldots) .
\end{array}\right.
$$

In fact, for each $n, F_{n}(r, \lambda, u)$ is strongly continuous on $[0, \infty) \times \mathbf{C}$ and the estimation 


$$
\left\|F_{n}(r, \lambda, u)\right\|_{H \leqq} \leqq \begin{array}{ll}
\frac{e^{b r}}{n !}\left(\frac{1}{|\sqrt{\lambda}|} \int_{0}^{r}\|A(t)\| d t\right)^{n}\|u\|_{H} & \text { if } \lambda \neq 0 \\
\frac{1}{n !}\left(r \int_{0}^{r}\|A(t)\| d t\right)^{n}\left\|_{i} u\right\|_{H} & \text { if } \lambda=0,
\end{array}
$$

hold, and hence $\sum_{n=0}^{\infty} F_{n}(r, \lambda, u)$ converges in $H$ uniformly on every finite interval in $[0, \infty)$. Therefore $F(r, \lambda, u)=\sum_{n=0}^{\infty} F_{n}(r, \lambda, u)$ is an $H$-valued, strongly continuous function on $[0, \infty) \times \mathbf{C}$. We can easily show that $F(r, \lambda, u)$ satisfies the integral equation (1.25) and that we have

$$
F(0, \lambda, u)=u \text {. }
$$

Moreover from (1.28) we obtain the estimation

$$
\begin{cases}\|F(r, \lambda, u)\|_{H} \leqq e^{g(b, r)}\|u\|_{H} & \\ g(b, r)= \begin{cases}b r+\frac{1}{|\sqrt{\lambda}|} \int_{0}^{r}\|A(t)\| d t & (\lambda \neq 0) \\ r \int_{0}^{r}\|A(t)\| d t & (\lambda=0)\end{cases} \end{cases}
$$

and taking note of the uniqueness of $F(r \lambda, u)$ we have

$$
F\left(r, \lambda, u_{1}+u_{2}\right)=F\left(r, \lambda, u_{1}\right)+F\left(r, \lambda, u_{2}\right),
$$

where $u_{1}$ and $u_{2}$ are arbitrary elements of $H$.

We can define a $\mathbf{B}$-valued function on $[0, \infty) \times \mathbf{C}, x(r, \lambda)$, by

$$
x(r, \lambda) u=F(r, \lambda, u) .
$$

In fact it follows from (1.28) and (1.30) that

$$
x(r, \lambda)=\sum_{n=0}^{\infty} x_{n}(r, \lambda)
$$

where

$$
\left\{\begin{array}{l}
x_{0}(r, \lambda)=\cos \sqrt{\lambda} r \cdot I \\
x_{n}(r, \lambda)=\int_{0}^{r} \frac{\sin \sqrt{\lambda}(r-t)}{\sqrt{\lambda}} A(t) x_{n-1}(t, \lambda) d t \quad(n=1,2,3 \ldots)
\end{array}\right.
$$

which are estimated as follows: 


$$
\begin{cases}\left\|x_{n}(r, \lambda)\right\| \leqq & \begin{cases}\frac{e^{b r}}{n !}\left(\frac{1}{|\sqrt{\lambda}|} \int_{0}^{r}\|A(t)\| d t\right)^{n}, & \text { if } \lambda \neq 0 \\ \frac{1}{n !}\left(r \int_{0}^{r}\|A(t)\| d t\right)^{n}, & \text { if } \lambda=0\end{cases} \\ \|x(r, \lambda)\| \leqq e^{g(b, r)} & \end{cases}
$$

Accordingly, taking account of (1.31), we can see that $x(r, \lambda)$ is a $\mathbf{B}$ valued function on $[0, \infty) \times \mathbf{C}$. For each $n, x_{n}(r, \lambda)$ is continuous on $[0, \infty) \times \mathbf{C}$ in the uniform topology of $\mathbf{B}$ and for any fixed $r \in[0, \infty)$ $x_{n}(r, \lambda)$ is a $\mathbf{B}$-valued, entire function. Therefore $x(r, \lambda)$ is continuous on $[0, \infty) \times \mathbf{C}$ in the uniform topology of $\mathbf{B}$ and for any $r \in[0, \infty) x(r, \lambda)$ is an entire function. We obtain from (1.25) and (1.29)

$$
\left\{\begin{array}{l}
x(r, \lambda)=\cos \sqrt{\lambda} r \cdot I+\int_{0}^{r} \frac{\sin \sqrt{\lambda}(r-t)}{\sqrt{\lambda}} A(t) x(t, \lambda) d t \\
x(0, \lambda)=I .
\end{array}\right.
$$

(II) Put

$$
y(r, \lambda)=-\sqrt{\lambda} \sin \sqrt{\lambda} r \cdot I+\int_{0}^{r} \cos \sqrt{\lambda}(r-t) A(t) x(t, \lambda) d t .
$$

We can easily show that $y(r, \lambda)$ is a $\mathbf{B}$-valued, continuous function on $[0, \infty) \times \mathbf{C}$ in the uniform topology of $\mathbf{B}$ and for any fixed $r \in[0, \infty)$ $y(r, \lambda)$ is a $\mathbf{B}$-valued, entire function. We have

$$
\begin{aligned}
& \frac{1}{h}\{x(r+h, \lambda)-x(r, \lambda)\}-y(r, \lambda) \\
= & \left\{\frac{1}{h}(\cos \sqrt{\lambda}(r+h)-\cos \sqrt{\lambda} r)+\sqrt{\lambda} \sin \sqrt{\lambda} r\right\} I \\
+ & \int_{0}^{r}\left(\frac{\sin \sqrt{\lambda}(r+h-t)-\sin \sqrt{\lambda}(r-t)}{h \sqrt{\lambda}}-\cos \sqrt{\lambda}(r-t)\right) A(t) x(t, \lambda) d t \\
+ & \int_{r}^{r+h} \frac{\sin \sqrt{\lambda}(r+h-t)}{h \sqrt{\lambda}} A(t) x(t, \lambda) d t=J_{1}+J_{2}+J_{3} .
\end{aligned}
$$

It is easy to see that

$$
\lim _{h \rightarrow 0}\left\|J_{1}\right\|=\lim _{h \rightarrow 0}\left\|J_{2}\right\|=0
$$

For $J_{3}$ we have 


$$
\begin{aligned}
\left\|J_{3}\right\| & \leqq \int_{r-|h|}^{r+|h|} \frac{1}{|h|}\left|\int_{0}^{r+h-t} \cos \sqrt{\lambda} s d s\right|\left\|\left.A(t)\right|_{i 1} \mid x(t, \lambda)\right\| d t \\
& \leqq 2 e^{b|h|} \int_{r-|h|}^{r+|h|}\|A(t)\|\|x(t, \lambda)\| d t \rightarrow 0 \quad(h \rightarrow 0),
\end{aligned}
$$

where $b=|\operatorname{Im} \sqrt{\lambda}|$ and we have made use of the estimations

$$
\left|\frac{\sin \sqrt{\lambda}(r+h-t)}{\sqrt{\lambda}}\right|=\left|\int_{0}^{r+h-t} \cos \sqrt{\lambda} s d s\right| \leqq|r+h-t| e^{b|r| h-t \mid}
$$

and

$$
|r+h-t| \leqq 2|h| \quad(t \in[r-|h|, r+|h|]) .
$$

Therefore we have for all $r \in[0, \infty)$

$$
\lim _{h \rightarrow 0}\left\|\frac{1}{h}(x(r+h, \lambda)-x(r, \lambda))-y(r, \lambda)\right\|=0,
$$

which implies that for each $\lambda \in \mathbf{C} \quad x(r, \lambda)$ is continuously differentiable on $[0, \infty)$ in the uniform topology of $\mathbf{B}$ with its derivative $x^{\prime}(r, \lambda)$ $=y(r, \lambda)$. Let $h>0$ and $u, v \in H$. Then we have

$$
\begin{aligned}
& \left(\left\{x^{\prime}(r+h, \lambda)-x^{\prime}(r, \lambda)\right\} u, v\right)_{H} \\
& =((-\sqrt{\lambda} \sin \sqrt{\lambda}(r+h)+\sqrt{\lambda} \sin \sqrt{\lambda} r) u, v) \\
& \quad+\int_{0}^{r}(\cos \sqrt{\lambda}(r+h-t)-\cos \sqrt{\lambda}(r-t)) \\
& \quad+\int_{0}^{r}\{\cos \sqrt{\lambda}(r+h-t) \cos \sqrt{\lambda}(r-t)\}(A(t) x(t, \lambda) u, v)_{H} d t \\
& \quad+\int_{r}^{r+h} \cos \sqrt{\lambda}(r+h-t)(A(t) x(t, \lambda) u, v)_{H} d t \\
& =K_{1}+K_{2}+K_{3} .
\end{aligned}
$$

We can estimate $K_{i}(i=1,2,3)$ as follows:

$$
\left\{\begin{aligned}
\left|K_{1}\right| & \leqq\left|\int_{r}^{r+h} \cos \sqrt{\lambda} d t\right|\|u\|\|v\| \leqq \int_{r}^{r+h} e^{b t} d t\|u\|\|v\| \\
\left|K_{2}\right| & \leqq \int_{0}^{r}\left|\int_{0}^{h} \frac{\sin \sqrt{\lambda}(r-t+s)}{\sqrt{\lambda}} d s\right|\|A(t) \mid i\| x(t, \lambda)\|d t\| u\|\| v \| \\
& \leqq h \int_{0}^{r} \frac{1}{|\sqrt{\lambda}|} e^{b(r-t+h)}\|A(t)\|\|x(t, \lambda)\| d t\|u\| !\|v\| \\
\left|K_{3}\right| & \leqq \int_{r}^{r+h} e^{2 b h}\|A(t)\|\|x(t, \lambda)\| d t\|u\|\|v\|
\end{aligned}\right.
$$


and hence $x^{\prime}(r, \lambda)$ is weakly absolutely continuous on every finite interval in $[0, \infty)$ for each $\lambda \in \mathbf{C}$. We obtain from (1.37)

$$
x^{\prime}(0, \lambda)=0
$$

(III) We denote $(A(r)-\lambda) x(r, \lambda)$ by $z(r, \lambda)$. Then $\|z(r, \lambda)\|$ is locally integrable on $[0, \infty)$ and for any fixed $r \in[0, \infty) z(r, \lambda)$ is a B-valued, entire function. For $u, v \in H$ we have

$$
\begin{aligned}
& \left(\frac{1}{h}\left(x^{\prime}(r+h, \lambda)-x^{\prime}(r, \lambda)\right) u, v\right)_{H}-(z(r, \lambda) u, v) \\
= & \left\{\frac{1}{h}(-\sqrt{\lambda} \sin \sqrt{\lambda}(r+h)+\sqrt{\lambda} \sin \sqrt{\lambda} r)+\lambda \cos \sqrt{\lambda} r\right\}(u, v)_{H} \\
+ & \int_{0}^{r}\left\{\frac{1}{h}(\cos \sqrt{\lambda}(r+h-t)-\cos \sqrt{\lambda}(r-t))\right. \\
& +\sqrt{\lambda} \sin \sqrt{\lambda}(r-t)\}(A(t) x(t, \lambda) u, v)_{H} d t \\
& +\int_{r}^{r+h} \frac{1}{h}(\cos \sqrt{\lambda}(r+h-t)-1)(A(t) x(t, \lambda) u, v)_{H} d t \\
& +\frac{1}{h} \int_{r}^{r+h}(A(t) x(t, \lambda) u, v)_{H} d t-(A(r) x(r, \lambda) u, v)_{H} \\
= & L_{1}+L_{2}+L_{3}+L_{4} .
\end{aligned}
$$

Estimations similar to the ones used in proving $\lim _{h \rightarrow 0}\left\|J_{i}\right\|=0(i=1,2,3)$ give

$$
\lim _{h \rightarrow 0}\left|L_{i}\right|=0 \quad(i=1,2,3)
$$

for all $r \in[0, \infty)$ and $u, v \in H$. Since $(A(t) x(t, \lambda) u, v)_{H}$ is locally integrable, there exists a null set $e(u, v, \lambda)$ in $[0, \infty)$ such that

$$
\lim _{h \rightarrow 0} \frac{1}{h} \int_{r}^{r+h}(A(t) x(t, \lambda) u, v)_{H} d t=(A(r) x(r, \lambda) u, v)_{H}
$$

holds for any $r \in[0, \infty)-e(u, v, \lambda)$, which implies that

$$
\lim _{h \rightarrow 0}\left|L_{4}\right|=0
$$


for all $r \in[0, \infty)-e(u, v, \lambda)$. Therefore we have

$$
\frac{d}{d r}\left(x^{\prime}(r, \lambda) u, v\right)=(z(r, \lambda) u, v)
$$

for all $r \in[0, \infty)-e(u, v, \lambda)$. It follows from Remark 1.5 that for any $u \in H$ and $\lambda \in \mathbf{C}$ we can choose a null set $e(u, \lambda)$ in $[0, \infty)$ such that

$$
\frac{d}{d r}\left(x^{\prime}(r, \lambda) u, v\right)=(z(r, \lambda) u, v)
$$

holds for all $v \in H$ and $r \in[0, \infty)-e(u, \lambda)$. Proceeding as in the proof of Lemma 1.4, we can also show that there exists a null set $e(\lambda)$ in $[0, \infty)$ such that $(1.51)$ is valid for all $u, v \in H$ and $r \in[0, \infty)-e(\lambda)$. Let $\left\{\lambda_{n}\right\}_{n=1}^{\infty}$ be a countable subset of $\mathbf{C}$ which is dense in $\mathbf{C}$. We put $e_{n}=e\left(\lambda_{n}\right)(n=1,2, \ldots)$ and denote by $e_{0}$ a null set in $[0, \infty)$ such that for all $r \in[0, \infty)-e_{0}$

$$
\frac{d}{d r}\left[\int^{r}\|A(t)\| d t\right]=\|A(r)\|
$$

is valid. Let $e=\bigcup_{m=0}^{\infty} e_{m}$ and let $r \in[0, \infty)-e$. Then we have for any $u, v \in H$ and $\lambda \in \mathbf{C}$

$$
\begin{aligned}
& \left(\left\{\frac{1}{h}\left(x^{\prime}(r+h, \lambda)-x^{\prime}(r, \lambda)\right)-z(r, \lambda)\right\} u, v\right)_{H} \\
= & \left(\left\{\frac{1}{h}\left(x^{\prime}\left(r+h, \lambda_{m}\right)-x^{\prime}\left(r, \lambda_{m}\right)\right)-z\left(r, \lambda_{m}\right)\right\} u, v\right)_{H} \\
+ & \left(\frac{1}{h}\left\{x^{\prime}(r+h, \lambda)-x^{\prime}(r, \lambda)\right\}-\frac{1}{h}\left\{x^{\prime}\left(r+h, \lambda_{m}\right)-x^{\prime}\left(r, \lambda_{m}\right)\right\} u, v\right)_{H} \\
& +\left(\left\{z\left(r, \lambda_{m}\right)-z(r, \lambda)\right\} u, v\right)_{H} \\
= & M_{1}+M_{2}+M_{3} .
\end{aligned}
$$

Noting $r \notin e_{m}$, we have $\lim _{h \rightarrow 0}\left|M_{1}\right|=0$. For $M_{2}$, we have

$$
\begin{gathered}
\left|M_{2}\right|=\left|\frac{1}{h} \int_{r}^{r+h}\left(\left\{z(t, \lambda)-z\left(t, \lambda_{m}\right)\right\} u, v\right)_{H} d t\right| \\
\leqq \frac{1}{|h|} \int_{r-|h|}^{r+|h|}\|A(t)\| d t \cdot\left(\max _{r-|h| \leqslant s \leqslant r+|h|}\left\|x(s, \lambda)-x\left(s, \lambda_{m}\right)\right\|\right) \\
\times\|u\|_{H}\|v\|_{H}
\end{gathered}
$$




$$
+\frac{1}{|h|} \int_{r-|h|}^{r_{\top}|h|}\left\|\lambda_{m} x\left(t, \lambda_{m}\right)-\lambda x(t, \lambda)\right\| d t\|u\|_{\mid H}\left\|_{i} v\right\|_{H}
$$

Since $r \in e_{0}$ and $x(r, \lambda)$ is continuous on $[0, \infty) \times \mathbb{C}$, it is obvious that $\lim _{\lambda_{m \rightarrow \lambda} \rightarrow \lambda}\left|M_{2}\right|=0$ and the convergence is uniform when $h$ tends to 0 . We can easily show that $\lim _{\lambda_{m} \rightarrow}\left\|z(r, \lambda)-z\left(r, \lambda_{m}\right)\right\|=0$, and hence $\lim \left|M_{3}\right|=0$. Thus we have proved that (1.51) is valid for all $u, v \in H, \lambda \in \mathbf{C}$ and $r \in[0, \infty)$ $-e$, where the null set $e$ does not depend on $u, v \in H$ and $\lambda \in \mathbf{C}$. Therefore for $r \in[0, \infty)-e x^{\prime}(r, \lambda)$ is weakly differentiable with its derivative $x^{\prime \prime}(r, \lambda)=z(r, \lambda)$.

(IV) It is easy to see from (I), (II) and (III) that $x(r, \lambda)$ satisfies (2) $\sim(5)$ of Proposition 1.4 and that $x(r, \lambda) u$ is the solution of (1.3) with data $\{u, 0\}$ for any $u \in H$. Consequently we have

$$
x(r, \lambda)=\omega(r, \lambda ; I, 0) .
$$

Thus we have completely proved the existence of $\omega(r, \lambda ; I, 0)$. In quite a similar way we can prove the existence of $\omega(r, \lambda ; I, 0)$, and by (1.24) the existence of $\omega(r, \lambda ; S, T)$ is shown.

Q. E. D.

Using Proposition 1.6, we give the following

Definition 1.8. Let $H_{1}$ and $H_{2}$ be closed, linear subspaces of $H$ such that

$$
H=H_{1} \oplus H_{2} \quad \text { (direct sum) }
$$

and let $B_{2}$ and $C_{2}$ be bounded, self-adjoint operators on $\mathrm{H}_{2}$ into itself such that

$$
B_{2} C_{2}=C_{2} B_{2}
$$

and the bounded inverse of $B_{2}$ exists. Then, putting

$$
B=\left\{\begin{array}{l}
0 \text { on } H_{1} \\
B_{2} \text { on } H_{2},
\end{array} \quad C=\left\{\begin{array}{l}
\text { identity on } H_{1} \\
C_{2} \text { on } H_{2},
\end{array}\right.\right.
$$

we define a bounded, linear operator $\phi(r, \lambda)$ by 


$$
\phi(r, \lambda)=\omega(r, \lambda ; B, C) .
$$

We can easily show that for each $\lambda \in \mathbf{C}$

$$
B \phi^{\prime}(0, \lambda)-C \phi(0, \lambda)=0 .
$$

Thus we have constructed the "eigenoperator" $\phi(r, \lambda)$ associated with $\mathscr{L}$ and the boundary condition

$$
B F^{\prime}(0)-C F(0)=0 .
$$

Here we remark that the boundary condition (1.62) is equivalent to

$$
\left\{\begin{array}{l}
P_{1} F(0)=0 \\
P_{2} F^{\prime}(0)=B_{2}^{-1} C_{2} P_{2} F(0),
\end{array}\right.
$$

where $P_{1}$ and $P_{2}$ are the projection operators onto $H_{1}$ and $H_{2}$, respectively.

We shall show the properties of $\frac{\partial \omega(r, \lambda)}{\partial \lambda}$ which will be used in $\S 4$.

Proposition 1.9. Let $\omega(r, \lambda)=\omega(r, \lambda ; S, T)$ be as in Proposition 1.6. Then we have following $(1) \sim(3)$ :

(1) For each $\lambda \in \mathbf{C}$ and $u \in H \frac{\partial \omega(r, \lambda)}{\partial \lambda} u$ belongs to $\mathbf{D}$.

(2) $\frac{\partial \omega(r, \lambda)}{\partial \lambda}$ is continuously differentiable on $[0, \infty)$ in the uniform topology of $\mathbf{B}$ with its derivative $\left(\frac{\partial \omega(r, \lambda)}{\partial \lambda}\right)^{\prime} \in \mathbf{B}$, and we have

$$
\left(\frac{\partial \omega(r, \lambda)}{\partial \lambda}\right)^{\prime}=\frac{\partial \omega^{\prime}(r, \lambda)}{\partial \lambda}
$$

on $[0, \infty) \times \mathbf{C}$.

(3) For each $\lambda \in \mathbf{C}\left(\frac{\partial \omega(r, \lambda)}{\partial \lambda}\right)$ ' is weakly absolutely continuous on every finite interval in $[0, \infty)$. There exists a null set $e$ in $[0, \infty)$ such that for any $r \in[0, \infty)-e\left(\frac{\partial \omega(r, \lambda)}{\partial \lambda}\right)$ ' is weakly differentiable with its derivative $\left(\frac{\partial \omega(r, \lambda)}{\partial \lambda}\right) " \in \mathbf{B}$. The exceptional set $e$ does not depend on $\lambda \in \mathbf{C} .\left\|\left(\frac{\partial \omega(r, \lambda)}{\partial \lambda}\right)^{\prime \prime}\right\|$ is locally integrable on $[0, \infty)$ and we have for all $r \in[0, \infty)-e$ and $\lambda \in \mathbf{C}$

$$
\left(\frac{\partial \omega(r, \lambda)}{\partial \lambda}\right)^{\prime \prime}=\frac{\partial \omega^{\prime \prime}(r, \lambda)}{\partial \lambda}
$$


and

$$
-\left(\frac{\partial \omega(r, \lambda)}{\partial \lambda}\right)^{\prime \prime}+A(r) \frac{\partial \omega(r, \lambda)}{\partial \lambda}=\lambda \frac{\partial \omega(r, \lambda)}{\partial \lambda}+\omega(r, \lambda) .
$$

Proof. Proposition 1.9 can be proved in a way similar to the proof of Proposition 1.6. Hence we shall give the outline of the proof. It is sufficient that we prove $(1) \sim(3)$ assuming $\omega(r, \lambda)=\omega(r, \lambda ; I, 0)$. Differentiating the both sides of (1.36) with respect to $\lambda$, we have

$$
\begin{aligned}
& \frac{\partial \omega(r, \lambda)}{\partial \lambda}=-\frac{r}{2 \sqrt{\lambda}} \sin \sqrt{\lambda} r \cdot I+\int_{0}^{r} \frac{\sin \sqrt{\lambda}(r-t)}{\sqrt{\lambda}} A(t) \frac{\partial \omega(t, \lambda)}{\partial \lambda} d t \\
& +\int_{0}^{r}\left\{\frac{r-t}{2 \lambda} \cos \sqrt{\lambda}(r-t)-\frac{\sin \sqrt{\lambda}(r-t)}{2 \lambda \sqrt{\lambda}}\right\} A(t) \omega(t, \lambda) d t .
\end{aligned}
$$

It follows from (1.67) that $\frac{\partial \omega(r, \lambda)}{\partial \lambda}$ is differentiable in the uniform topology for each $\lambda \in \mathbf{C}$ and that we have

$$
\begin{aligned}
\left(\frac{\partial \omega(r, \lambda)}{\partial \lambda}\right)^{\prime}= & \left(-\frac{1}{2 \sqrt{\lambda}} \sin \sqrt{\lambda}(r-t)-\frac{r}{2} \cos \sqrt{\lambda} r\right) I \\
& +\int_{0}^{r} \cos \sqrt{\lambda}(r-t) A(t) \frac{\partial \omega(t, \lambda)}{\partial \lambda} d t \\
& -\int_{0}^{r} \frac{(r-t)}{2 \sqrt{\lambda}} \sin \sqrt{\lambda}(r-t) A(t) \omega(t, \lambda) d t
\end{aligned}
$$

On the other hand we obtain from (1.37),

$$
\begin{aligned}
\frac{\partial \omega^{\prime}(r, \lambda)}{\partial \lambda}= & \frac{\partial}{\partial \lambda}\left\{-\sqrt{\lambda} \sin \sqrt{\lambda} r \cdot I+\int_{0}^{r} \cos \sqrt{\lambda}(r-t) A(t) \omega(t, \lambda) d t\right\} \\
= & \left(-\frac{1}{2 \sqrt{\lambda}} \sin \sqrt{\lambda} r-\frac{r}{2} \cos \sqrt{\lambda} r\right) I \\
& +\int_{0}^{r} \cos \sqrt{\lambda}(r-t) A(t) \frac{\partial \omega(t, \lambda)}{\partial \lambda} d t \\
& -\int_{0}^{r} \frac{r-t}{2 \sqrt{\lambda}} \sin \sqrt{\lambda}(r-t) A(t) \omega(t, \lambda) d t,
\end{aligned}
$$

and hence we have $\left(\frac{\partial \omega(r, \lambda)}{\partial \lambda}\right)^{\prime}=\frac{\partial \omega^{\prime}(r, \lambda)}{\partial \lambda}$. Thus (2) has been proved. It is easy to see that $\frac{\partial \omega^{\prime}(r, \lambda)}{\partial \lambda}$ is weakly absolutely continuous 
on every finite interval in $[0, \infty)$. Let $u, v \in H$. Then we have

$$
\begin{aligned}
& \frac{1}{h}\left(\left\{\frac{\partial \omega^{\prime}(r+h, \lambda)}{\partial \lambda}-\frac{\partial \omega^{\prime}(r, \lambda)}{\partial \lambda}\right\} u, v\right)_{H} \\
& =\frac{\partial}{\partial \lambda}\left(\frac{\omega^{\prime}(r+h, \lambda)-\omega^{\prime}(r, \lambda)}{h} u, v\right)_{H} \\
& =\frac{\partial}{\partial \lambda}\left\{\frac{1}{h} \int_{r}^{r+h}\left(\omega^{\prime \prime}(t, \lambda) u, v\right)_{H} d t\right\} \\
& =\frac{\partial}{\partial \lambda}\left\{\frac{1}{h} \int_{r}^{r+h}((A(t)-\lambda) \omega(t, \lambda) u, v)_{H} d t\right\} .
\end{aligned}
$$

Here we have made use of the fact that $\omega^{\prime \prime}(r, \lambda)=(A(r)-\lambda) \omega(r, \lambda)$. Let $N$ be an arbitrary positive number. Then the integral

$$
\begin{aligned}
& \int_{0}^{N}\left|\frac{\partial}{\partial \lambda}((A(t)-\lambda) \omega(t, \lambda) u, v)_{H}\right| d t \\
& \quad=\int_{0}^{N}\left|\left(\left\{(A(t)-\lambda) \frac{\partial \omega(t, \lambda)}{\partial \lambda}-\omega(t, \lambda)\right\} u, v\right)_{H}\right| d t
\end{aligned}
$$

is continuous on C. Therefore we can interchange of the order of differentiation and integration in (1.70) to obtain

$$
\begin{aligned}
& \frac{1}{h}\left(\left\{\frac{\partial \omega^{\prime}(r+h, \lambda)}{\partial \lambda}-\frac{\partial \omega^{\prime}(r, \lambda)}{\partial \lambda}\right\} u, v\right)_{H} \\
& =\frac{1}{h} \int_{r}^{r+h}\left(\left\{(A(t)-\lambda) \frac{\partial \omega(t, \lambda)}{\partial \lambda}-\omega(t, \lambda)\right\} u, v\right)_{H} d t .
\end{aligned}
$$

It follows from (1.72) that

$$
\frac{d}{d r}\left(\frac{\partial \omega^{\prime}(r, \lambda)}{\partial \lambda} u, v\right)_{H}=\left(\left\{(A(r)-\lambda) \frac{\partial \omega(r, \lambda)}{\partial \lambda}-\omega(r, \lambda)\right\} u, v\right)_{H}
$$

holds almost everywhere. Using arguments similar to the ones in proving (4) of Proposition 1.6, we can easily show that we can choose a null set $\boldsymbol{e}$ in $[0, \infty)$ which does not depend on $u, v \in H$ and $\lambda \in \mathbf{C}$ such that for any $r \in[0, \infty)-e, \lambda \in \mathbf{C}$ and $u, v \in H$ (1.73) holds. And we have for almost all $r \in[0, \infty)$

$$
\left(\frac{\partial \omega^{\prime}(r, \lambda)}{\partial \lambda}\right)^{\prime}=(A(r)-\lambda) \frac{\partial \omega(r, \lambda)}{\partial \lambda}-\omega(r, \lambda)=\frac{\partial \omega^{\prime \prime}(r, \lambda)}{\partial \lambda},
$$


which completes the proof of (3). (1) is immediate from (2) and (3).

Q.E.D.

\section{§2. Green's Formula for $H$-valued Functions}

In this section we shall give Green's formula for $H$-valued functions which will be useful in $\S 3$.

Proposition 2.1 (Green's formula). Take H-valued functions $F_{1}(r)$ and $F_{2}(r)$ on $[0, \infty)$ such that $F_{i} \in \mathbf{D}(i=1,2)$. Take a fivite, closed interval $[a, \beta]$ in $[0, \infty)$. Then we have

$$
\begin{aligned}
\int_{\alpha}^{\beta}\left\{\left(\mathscr{L} F_{1}(r), F_{2}(r)\right)_{H}\right. & \left.-\left(F_{1}(r), \mathscr{L} F_{2}(r)\right)_{H}\right\} d r \\
= & {\left[F_{1}, F_{2}\right](\beta)-\left[F_{1}, F_{2}\right](\alpha), }
\end{aligned}
$$

where

$$
\mathscr{L} F_{i}(r)=-F_{i}^{\prime \prime}(r)+A(r) F_{i}(r) \quad(i=1,2),
$$

and

$$
\left[F_{1}, F_{2}\right](r)=\left(F_{1}(r), F_{2}^{\prime}(r)\right)_{H}-\left(F_{1}^{\prime}(r), F_{2}(r)\right)_{H}
$$

Proof. Since $F_{1}^{\prime}(r)$ and $F_{2}(r)$ are strongly absolutely continuous, ${ }^{5)}$ $\left(F_{1}^{\prime}(r), F_{2}(r)\right)_{H}$ is absolutely continuous on $[\alpha, \beta]$. We calculate $\frac{d}{d r}$ $\left(F_{1}^{\prime}(r), F_{2}(r)\right)_{H}$ as follows: we have

$$
\begin{aligned}
& \frac{1}{h}\left\{\left(F_{1}^{\prime}(r+h), F_{2}(r+h)\right)-\left(F_{1}^{\prime}(r), F_{2}(r)\right)_{H}\right\} \\
& =\left(\frac{F_{1}^{\prime}(r+h)-F_{1}^{\prime}(r)}{h}, F_{2}(r)\right)+\left(F_{1}^{\prime}(r), \frac{F_{2}(r+h)-F_{2}(r)}{h}\right) \\
& \quad+\left(F_{1}^{\prime}(r+h)-F_{1}^{\prime}(r), \frac{F_{2}(r+h)-F_{2}(r)}{h}\right)=J_{1}+J_{2}+J_{3} .
\end{aligned}
$$

Since $F_{1}^{\prime}(r)$ is weakly differentiable almost everywhere, we have

$$
\lim _{h \rightarrow 0} J_{1}=\left(F_{1}^{\prime \prime}(r), F_{2}(r)\right)
$$

5) See Lemma 1.3 in $\$ 1$. 
for almost all $r \in[\alpha, \beta]$ and it follows from the strong differentiability of $F_{2}(r)$ that

$$
\lim _{h \rightarrow 0} J_{2}=\left(F_{1}^{\prime}(r), F_{2}^{\prime}(r)\right)
$$

for all $r \in[\alpha, \beta]$. As for $J_{3}$, we have

$$
\left|J_{3}\right| \leqq\left\|F_{1}^{\prime}(r+h)-F_{1}^{\prime}(r)\right\|\left\|-\frac{F_{2}(r+h)-F_{2}(r)}{h}\right\| \text {. }
$$

Since $F_{2}^{\prime}(r)$ exists, $\left\|\frac{1}{h}\left(F_{2}(r+h)-F_{2}(r)\right)\right\|$ is bounded when $h$ tends to 0 . Hence

$$
\lim _{h \rightarrow 0}\left|J_{3}\right|=0
$$

for all $r \in[\alpha, \beta]$. Therefore we obtain for almost all $r \in[\alpha, \beta]$

$$
\frac{d}{d r}\left(F_{1}^{\prime}(r), F_{2}(r)\right)=\left(F_{1}^{\prime \prime}(r), F_{2}(r)\right)+\left(F_{1}^{\prime}(r), F_{2}^{\prime}(r)\right) .
$$

Integrating (2.9) we can show

(2.10) $\left.\quad\left(F_{1}^{\prime}(r), F_{2}(r)\right)_{H}\right|_{\alpha} ^{\beta}=\int_{\alpha}^{\beta}\left\{\left(F_{1}^{\prime \prime}(r), F_{2}(r)\right)_{H}+\left(F_{1}^{\prime}(r), F_{2}^{\prime}(r)\right)_{H}\right\} d r$.

Quite similarly we have

(2.11) $\left.\quad\left(F_{1}(r), F_{2}^{\prime}(r)\right)_{H}\right|_{\alpha} ^{\beta}=\int_{\alpha}^{\beta}\left\{\left(F_{1}(r), F_{2}^{\prime \prime}(r)\right)_{H}+\left(F_{1}^{\prime}(r), F_{2}^{\prime}(r)\right)_{H}\right\} d r$.

(2.1) is obtained from (2.10) and (2.11).

Q.E.D.

Corollary 2.2. Let $F_{1} \in \mathbf{D}$ and let $F_{2}$ satisfy (1) of Definition 1.2. Then we have

$$
\begin{aligned}
\int_{\alpha}^{\beta} & \left(\mathscr{L} F_{1}(r), F_{2}(r)\right)_{H} d r \\
& =\int_{\alpha}^{\beta}\left\{\left(F_{1}^{\prime}(r), F_{2}^{\prime}(r)\right)_{H}+\left(A(r) F_{1}(r), F_{2}(r)\right)_{H}\right\} d r \\
& -\left.\left(F_{1}^{\prime}(r), F_{2}(r)\right)\right|_{\alpha} ^{\beta}
\end{aligned}
$$

for $0 \leqq \alpha<\beta<\infty$.

Proof. Notice that it is not necessary to show (2.9) that $F_{2}$ satisfies (2) of Definition 1.2. Then (2.12) is obtained immediately from (2.10). 
Q.E.D.

\section{§3. The Operator $L$}

Let $\mathscr{L}$ be the differential operator defined in Definition 1.2. We consider a restriction $L$ of $\mathscr{L}$ which satisfies the boundary condition

$$
B F^{\prime}(0)-C F(0)=0,
$$

where $B$ and $C$ are bounded, self-adjoint operators on $H$ defined in Definition 1.8. When the differential operator $\mathscr{L}$ and the boundary condition (3.1) are given, an operator $L$ will be defined by $L F=\mathscr{L} F$ on the set of all functions $F(r)$ such that $F$ and $\mathscr{L} F$ are well-defined as elements of $\mathfrak{h}$ and $F$ satisfies (3.1). We shall prove that $L$ is a closed operator and that if $F_{n}(n=1,2, \ldots)$ belong to the domain of $L$ and both $F_{n}$ and $L F_{n}$ converge in $\mathfrak{h}$, then the sequence $F_{n}(r)$ and $F_{n}^{\prime}(r)$ also converge in $H$ for any $r \geqq 0$. These results will be used in $\S 4$.

Definition 3.1. Let $\mathscr{L}$ be as in Definition 1.2. Let us denote by $\mathscr{D}$ the set of all $H$-valued functions $F(r)$ on $[0, \infty)$ which satisfy the following condition:

(i) $F \in \mathbf{D}$.

(ii) We have

$$
B F^{\prime}(0)-C F(0)=0 \text {. }
$$

(iii) Both $F(r)$ and $\mathscr{L} F(r)=-F^{\prime \prime}(r)+A(r) F(r)$ belong to $\mathfrak{h}$. Then we define an operator $L$ in $\mathfrak{h}$ by

$$
\left\{\begin{array}{l}
\mathscr{B}(L)=\mathscr{D} \\
L F(r)=\mathscr{L} F(r) .
\end{array}\right.
$$

Theorem 3.2. Let $L$ be as in Definition 3.1. Then $L$ is a closed operator. Further, if $F_{n} \in \mathscr{D} \quad n=1,2,3 \cdots$ and two sequences $F_{n}$ and $L F_{n}$ are Cauchy sequences in $\mathfrak{h}$, then two sequences $F_{n}(r)$ and $F_{n}^{\prime}(r)$ are Cauchy sequences in $H$ and they converge uniformly on every finite interval in $[0, \infty) . \quad$ Put $F=\lim _{n \rightarrow \infty} F_{n}$ in $\mathfrak{h}$. Then there is a null set $e$ in $[0, \infty)$ such 
that we can modify $F(r)$ on $e$ to obtain

$$
F_{n}(r) \rightarrow F(r) \quad \text { in } H,
$$

and

$$
F_{n}^{\prime}(r) \rightarrow F^{\prime}(r) \quad \text { in } H
$$

for all $r \in[0, \infty)$.

In order to prove this theorem, we prepare several lemmas.

Lemma 3.3. Let $G_{n}$ be a sequence of $H$-valued functions on $[\alpha, \beta]$, where $[\alpha, \beta]$ is a finite interval in $[0, \infty)$, satisfying the following:

(a) For all $u \in H,\left(G_{n}(r), u\right)_{H}$ is absolutely continuous on $[\alpha, \beta]$ and $G_{n}$ is weakly differentiable almost everywhere with the weak derivative $G_{n}^{\prime}$ on $[\alpha, \beta]$.

(b) Both $G_{n}$ and $G_{n}^{\prime}$ are Cauchy sequences in $L_{1}(\alpha, \beta ; H)$. Then the sequence $G_{n}(r)$ converges uniformly in $H$ on $[\alpha, \beta]$.

Proof. We show that $G_{n}(r)$ converges uniformly on $\left[\frac{\alpha+\beta}{2}, \beta\right]$. Take a smooth function $p(t)$ on $[\alpha, \beta]$ such that $p(t)=1$ for $t \in$ $\left[\frac{\alpha+\beta}{2}, \beta\right]$ and $p(t)=0$ for $t \in\left[\alpha, \frac{\alpha+\beta}{3}\right]$. Then for all $u \in H$ and $r \in\left[\frac{\alpha+\beta}{2}, \beta\right]$

$$
\begin{aligned}
\left(G_{n}(r)\right. & \left.-G_{m}(r), u\right)_{H}=\int_{\alpha}^{r} \frac{d}{d t}\left[p(t)\left(G_{n}(t)-G_{m}(t), u\right)_{H}\right] d t \\
& =\int_{\alpha}^{r}\left\{p^{\prime}(t)\left(G_{n}(t)-G_{m}(t), u\right)+p(t)\left(G_{n}^{\prime}(t)-G_{m}^{\prime}(t), u\right)\right\} d t .
\end{aligned}
$$

From (3.6) we obtain

$$
\left\|G_{n}(r)-G_{m}(r) i\right\|_{H} \leqq C(p)\left\{\int_{\alpha}^{\beta}\left\|G_{n}(t)-G_{m}(t)\right\| d t+\int_{\alpha}^{\beta}\left\|G_{n}^{\prime}(t)-G_{m}^{\prime}(t)\right\| d t\right\},
$$

where $C(p)$ is a positive constant depending only on $p(t)$. Since $G_{n}$ and $G_{n}^{\prime}$ are Cauchy sequences in $L_{1}(\alpha, \beta ; H)$, it follows from (3.7) that $\lim _{m, n \rightarrow \infty}\left\|G_{n}(r)-G_{m}(r)\right\|_{H}=0$ and the sequence $G_{n}(r)$ converges uniformly on $\left[\frac{\alpha+\beta}{2}, \beta\right]$. Similarly we can show that $G_{n}(r)$ converges uniformly on 
$\left[\alpha,-\frac{\alpha+\beta}{2}\right]$

Q.E. D.

Using Green's formula we obtain

Lemma 3.4. Let $F \in \mathscr{D}$ and let $p(r)$ be a smooth function on $[0, \infty)$ with a compact carrier in $[0, \infty)$. Then we have

$$
\begin{aligned}
\left\|p F^{\prime}\right\|_{h}^{2}= & (p W, p F)_{\natural}-2\left(p F^{\prime}, p^{\prime} F\right)_{\text {G }}-(A p F, p F)_{\mathfrak{G}} \\
& -\left(p(0) B_{2}^{-1} C_{2} P_{2} F(0), p(0) F(0)\right)_{H}
\end{aligned}
$$

where $W=L F, B_{2}$ and $C_{2}$ are as in Definition 1.8 and $P_{2}$ is the projection operator onto $\mathrm{H}_{2}$.

Proof. Let $p(r)=0$ for $r \geqq R$ and $G \in \mathscr{D}$. Putting $F_{1}=F$ and $F_{2}=$ $p G$ and $[\alpha, \beta]=[0, R]$ in the formula (2.12) of Corollary 2.2. we obtain

$$
(L F, p G)_{\mathfrak{h}}=\left(F^{\prime},(p G)^{\prime}\right)_{\mathfrak{h}}+(A F, p G)_{\mathfrak{G}}+\left(F^{\prime}(0), p(0) G(0)\right)_{H},
$$

which is rewritten in the form

$$
\begin{aligned}
\left(p F^{\prime}, G^{\prime}\right)_{\mathfrak{h}} & =(p W, G)_{\mathfrak{h}}-\left(p^{\prime} F^{\prime}, G\right)_{\mathfrak{h}} \\
& -(A p F, G)_{\mathfrak{h}}-\left(p(0) F^{\prime}(0), G(0)\right)_{H} .
\end{aligned}
$$

Putting $G=p F$ in (3.9) we have

$$
\begin{aligned}
\left(p F^{\prime}, p F^{\prime}\right)_{\mathfrak{h}}=(p W, p F)_{\mathfrak{h}}-2\left(p F^{\prime}, p^{\prime} F\right)_{\mathfrak{h}}-(A p F, p F)_{\mathfrak{h}} & \\
& -\left(p(0) F^{\prime}(0), p(0) F(0)\right)_{H} .
\end{aligned}
$$

Since $F \in \mathscr{D}, F$ satisfies the boundary condition (3.1) which is equivalent to

$$
\left\{\begin{array}{l}
P_{1} F(0)=0 \\
P_{2} F^{\prime}(0)=B_{2}^{-1} C_{2} P_{2} F(0) .^{6)}
\end{array}\right.
$$

Therefore we obtain

$$
\left(p(0) F^{\prime}(0), p(0) F(0)\right)_{H}=\left(p(0) B_{2}^{-1} C_{2} P_{2} F(0), p(0) F(0)\right)_{H},
$$

and (3.8) is obtained from (3.10) and (3.12).

Q.E. D.

Using Lemma 3.3 and Lemma 3.4 we prove

6) See (1.63) in $\$ 1$. 
Lemma 3.5. Let $F_{n}$ be as in Theorem 3.2. Then there exists an $H$-valued function $V(r)$ on $(0, \infty)$ such that $F_{1}^{\prime} \rightarrow V$ in $L_{2}(\alpha, \beta ; H)$ for any positive numbers $\alpha, \beta(\alpha<\beta)$, and $F_{n}(r)$ is a Canchy sequence in $H$ for any $r>0$.

Proof. Take a smooth function $p(r)$ such that $0 \leqq p \leqq 1, p(r)=0$ for $0 \leqq r \leqq \frac{\alpha}{2}$ and $r \geqq \beta+1$ and $p(r)=1$ for $\alpha \leqq r \leqq \beta$. Put $F=F_{n}-F_{m}$ and $W=W_{n}-W_{m}$, where $W_{n}=L F_{n}$. Since $F_{n}-F_{m} \in \mathscr{D}$ we can use Lemma 3.4 to obtain

$$
\begin{aligned}
\left\|p\left(F_{n}^{\prime}-F_{m}^{\prime}\right)\right\|_{\mathfrak{h}}^{2} & =\left(p\left(W_{n}-W_{m}\right), p\left(F_{n}-F_{m}\right)\right)_{\mathfrak{h}} \\
& -2\left(p\left(F_{n}^{\prime}-F_{m}^{\prime}\right), p^{\prime}\left(F_{n}-F_{m}\right)\right)_{\mathfrak{\eta}} \\
& -\left(A p\left(F_{n}-F_{m}\right), p\left(F_{n}-F_{m}\right)\right)_{\mathfrak{h}} .
\end{aligned}
$$

Let us estimate the term- $\left(A p\left(F_{n}-F_{m}\right), p\left(F_{n}-F_{m}\right)\right)_{\mathfrak{y}}$. From (3) of Assumption 1.1 we obtain

$$
\begin{aligned}
& -\left(A p\left(F_{n}-F_{m}\right), p\left(F_{n}-F_{m}\right)\right)_{\mathfrak{h}} \\
& =-\int_{0}^{\beta+1}\left(A(r) p(r)\left(F_{n}(r)-F_{m}(r)\right), p(r)\left(F_{n}(r)-F_{m}(r)\right)\right)_{H} d r \\
& \quad \leq C_{\beta+1}\left\|p\left(F_{n}-F_{m}\right)\right\|_{\mathrm{h}}^{2} \leqq C_{\beta+1}\left\|F_{n}-F_{m}\right\|_{\mathrm{h} .}^{2} .
\end{aligned}
$$

It follows from (3.13) and (3.14) that

$$
\begin{aligned}
\left\|p\left(F_{n}^{\prime}-F_{m}^{\prime}\right)\right\|^{2} & \leqq\left\|W_{n}-W_{m}\right\|_{\mathfrak{h}}\left\|F_{n}-F_{m}\right\|_{\mathfrak{\zeta}} \\
& +2 C(p)\left\|p\left(F_{n}^{\prime}-F_{m}^{\prime}\right)\right\|_{\mathfrak{h}}\left\|F_{n}-F_{m}\right\|_{\mathfrak{\zeta}} \\
& +C_{\beta+1}\left\|F_{n}-F_{m}\right\|_{\mathfrak{h}}^{2},
\end{aligned}
$$

where $C(p)$ is a positive constant depending only on $p(r)$. Since $F_{n}$ and $W_{n}$ are Cauchy sequences in $\mathfrak{h}, p F_{n}^{\prime}$ becomes a Cauchy sequence in $\mathfrak{h}$, which implies that $F_{n}^{\prime}$ is a Cauchy sequence in $L_{2}(\alpha, \beta ; H)$. Therefore we have proved the existence of $V(r)$ satisfying $F_{n}^{\prime} \rightarrow V$ in $L_{2}(\alpha, \beta ; H)$. Applying Lemma 3.3, putting $G_{n}=F_{n}$, we can see that $F_{n}(r)$ is a Cauchy sequence in $H$ for $r>0$. This finishes the proof.

Q. E. D.

In the following lemma we shall show that $F_{n}^{\prime}$ is really a Cauchy 
sequence in $L_{2}(0, \beta ; H)$.

Lemma 3.6. Let $F_{n}$ be as in Theorem 3.2 and let $V(r)$ be as in Lemma 3.5. Then $F_{n}^{\prime} \rightarrow V$ in $L_{2}(0, \beta ; H)$ and the sequence $F_{n}(r)$ converges uniformly on $[0, \beta]$ for any $\beta>0$.

Proof. Take a smooth function $p(r)$ on $[0, \infty)$ satisfying $0 \leqq p \leqq 1$, $p(r)=1$ for $0 \leqq r \leqq \beta$ and $p(r)=0$ for $r \geqq \beta+1$. It follows from Lemma 3.5 that there exists a positive number $r_{0} \leqq \beta$ such that $F_{n}\left(r_{0}\right)$ is a Cauchy sequence in $H$ and $2\left\|B_{1}^{-1}\right\|\|\| C_{2} \| r_{0}<1$. Let us estimate $\left\|F_{n}(0)-F_{m}(0)\right\|_{H}$. Using repeatedly the relation

$$
\begin{aligned}
\left(F_{n}(0)-F_{m}(0), u\right)= & \left(F_{n}\left(r_{0}\right)-F_{m}\left(r_{0}\right), u\right) \\
& -\int_{0}^{r_{0}}\left(p(r)\left(F_{n}^{\prime}(r)-F_{m}^{\prime}(r)\right), u\right) d r
\end{aligned}
$$

where $u \in H$, we have

$$
\begin{aligned}
& \left\|F_{n}(0)-F_{m}(0)\right\|_{H}^{2}=\left(F_{n}\left(r_{0}\right)-F_{m}\left(r_{0}\right), F_{n}(0)-F_{m}(0)\right)_{H} \\
& \quad \quad-\int_{0}^{r_{0}}\left(p(r)\left(F_{n}^{\prime}(r)-F_{m}^{\prime}(r)\right), F_{n}(0)-F_{m}(0)\right)_{H} d r \\
& =\left\|F_{n}\left(r_{0}\right)-F_{m}\left(r_{0}\right)\right\|^{2}-\int_{0}^{r_{0}}\left(F_{n}\left(r_{0}\right)-F_{m}\left(r_{0}\right), p(r)\left(F_{n}^{\prime}(r)-F_{m}^{\prime}(r)\right)\right)_{H} d r \\
& \quad-\int_{0}^{r_{0}}\left(p(r)\left(F_{n}^{\prime}(r)-F_{m}^{\prime}(r)\right), F_{n}\left(r_{0}\right)-F_{m}\left(r_{0}\right)\right)_{H} d r \\
& -\int_{0}^{r_{0}} \int_{0}^{r_{0}}\left(p(r)\left(F_{n}^{\prime}(r)-F_{n}^{\prime}(r)\right), p(t)\left(F_{n}^{\prime}(t)-F_{n}^{\prime}(t)\right)\right)_{H} d r d t .
\end{aligned}
$$

It follows from (3.17) that

$$
\begin{aligned}
& \left\|F_{n}(0)-\left.F_{m}(0)\right|^{2} \leqq\right\| F_{n}\left(r_{0}\right)-F_{m}\left(r_{0}\right) \|^{2} \\
& +2\left\|F_{n}\left(r_{0}\right)-F_{m}\left(r_{0}\right)\right\| \int_{0}^{r_{0}}\left\|p(t)\left(F_{n}^{\prime}(t)-F_{m}^{\prime}(t)\right)\right\| d t \\
& +\left[\int_{0}^{r_{0}}\left\|(t)\left(p F_{n}^{\prime}(t)-F_{m}^{\prime}(t)\right)\right\| d t\right]^{2} \\
& \leqq\left\|F_{n}\left(r_{0}\right)-\left.F_{m}\left(r_{0}\right)\right|_{H} ^{2}+2 \sqrt{r_{0}}\right\| F_{n}\left(r_{0}\right)-F_{m}\left(r_{0}\right)\left\|_{H}\right\| p\left(F_{n}^{\prime}-F_{m}^{\prime}\right) \|_{\xi} \\
& +r_{0}\left\|p\left(F_{n}^{\prime}-F_{m}^{\prime}\right)\right\|_{h}^{2}
\end{aligned}
$$

Proceeding as in the proof of Lemma 3.5, we have from (3.8), (3.14) and (3.18) 


$$
\begin{aligned}
& \left\|p\left(F_{n}^{\prime}-F_{m}^{\prime}\right)\right\|_{\eta}^{2} \\
& \leqq \\
& \quad+W_{n}-W_{m}\left\|_{\mathfrak{h}}\right\| F_{n}-F_{m}\left\|_{\mathfrak{h}}+2\right\| p\left(F_{n}^{\prime}-F_{m}^{\prime}\right)\left\|_{\mathfrak{h}}\right\| p^{\prime}\left(F_{n}-F_{m}\right) \|_{\mathfrak{h}} \\
& \quad+C_{\beta+1}\left\|F_{n}-F_{m}\right\|_{\mathfrak{h}}^{2}+\left\|B_{2}^{-1}\right\|\left\|C_{2}\right\|\left\{\left\|F_{n}\left(r_{0}\right)-F_{m}\left(r_{0}\right)\right\|_{H}^{2}\right. \\
& \left.\quad+2 \sqrt{r_{0}}\left\|F_{n}\left(r_{0}\right)-F_{m}\left(r_{0}\right)\right\|_{H}\left\|p\left(F_{n}^{\prime}-F_{m}^{\prime}\right)\right\|_{\mathfrak{h}}+r_{0}\left\|p\left(F_{n}^{\prime}-F_{m}^{\prime}\right)\right\|_{h}^{2}\right\} .
\end{aligned}
$$

As $\left\|B_{2}^{-1}\right\|_{\|}\left\|_{1} C_{2}\right\| r_{0}<1 / 2$, it follows from (3.19) that

$$
\begin{aligned}
& (3.20) \frac{1}{2}\left\|p\left(F_{n}^{\prime}-F_{m}^{\prime}\right)\right\|_{\mathfrak{h}}^{2} \\
& \leqq 2\left\{\left\|p^{\prime}\left(F_{n}-F_{m}\right)\right\|_{\mathfrak{h}}+\sqrt{r_{0}}\left\|B_{2}^{-1}\right\|\left\|C_{2}\right\|\left\|F_{n}\left(r_{0}\right)-F_{m}\left(r_{0}\right)\right\|_{H}\right\}\left\|p\left(F_{n}^{\prime}-F_{m}^{\prime}\right)\right\|_{\mathfrak{h}} \\
& \quad+\left\|W_{n}-W_{m}\right\|_{\mathfrak{h}}\left\|F_{n}-F_{m}\right\|_{\mathfrak{h}}+C_{\beta+1}\left\|F_{n}-F_{m}\right\|_{\mathfrak{h}}^{2} \\
& +\left\|B_{2}^{-1}\right\|\left\|C_{2}\right\|\left\|F_{n}\left(r_{0}\right)-F_{m}\left(r_{0}\right)\right\|_{H},
\end{aligned}
$$

which implies that $\lim _{n \rightarrow m}\left(F_{n}^{\prime}-F_{m}^{\prime}\right)=0$ in $L_{2}(0, \beta ; H)$. On the other hand the sequence $F_{n}$ is assumed to be a Cauchy sequence in $\mathfrak{h}$. Thus we have shown $F_{n}$ and $F_{n}^{\prime}$ are Cauchy sequences in $L_{2}(0, \beta ; H)$ for any $\beta>0$. Hence we can make use of Lemma 3.3 to see that the sequence $F_{n}(r)$ converges uniformly on $[0, \beta]$ Q. E. D.

Lemma 3.7. Let $F_{n}$ be as in Theorem 3.2. Then for any $\beta>0$ $F_{n}^{\prime \prime}$ is a Cauchy sequence in $L_{1}(0, \beta ; H)$ and $F_{n}^{\prime}(r)$ converges uniformly in $H$ on $[0, \beta]$.

Proof. Since $F_{n}^{\prime \prime}(r)=A(r) F_{n}(r)-W_{n}(r)$, we obtain

$$
\begin{aligned}
& \int_{0}^{\beta}\left\|F_{n}^{\prime \prime}(s)-F_{m}^{\prime \prime}(s)\right\|_{H} d s \\
& =\int_{0}^{\beta}\left\|A(s)\left(F_{n}(s)-F_{m}(s)\right)+\left(W_{m}(s)-W_{n}(s)\right)\right\|_{H} d s \\
& \left.\leqq \max _{0 \leqq s \leqq \beta}\left\|F_{n}(s)-F_{m}(s)\right\|_{H}\right) \times \int_{0}^{\beta}\|A(s)\| d s \\
& \quad+\int_{0}^{\beta}\left\|W_{m}(s)-W_{n}(s)\right\|_{H} d s,
\end{aligned}
$$

where $W_{n}=L F_{n}$. By (2) of Assumption $1.1\|A(r)\|$ is locally integrable, i.e.

$$
\int_{0}^{\beta} \mid l A(t) \| d t<\infty
$$

Moreover it follows from Lemma 3.6 that 


$$
\lim _{n, m \rightarrow \infty} \max _{0 \leqq s \leqq \beta}\left\|F_{n}(s)-F_{m}(s)\right\|_{H}=0 .
$$

From (3.21), (3.22) and (3.23) we see

$$
\int_{0}^{\beta} \| F_{n}^{\prime \prime}(s)-\left.F_{m}^{\prime \prime}(s)\right|_{H} d s \rightarrow 0 \quad(n, m \rightarrow \infty)
$$

Recalling that the sequence $F_{n}^{\prime}$ is a Cauchy sequence in $L_{2}(0, \beta ; H)$, we can use Lemma 3.3 to show that the sequence $F_{n}^{\prime}(r)$ converges uniformly on $[0, \beta]$.

Q. E. D.

Using Lemma 3.6 and Lemma 3.7, we can easily show

Lemma 3.8. Let $F$ and $F_{n}$ be as in Theorem 3.2 and let $V$ be as in Lemma 3.5. Then there exist H-valued functions $F_{0}(r)$ and $V_{0}(r)$ on $[0, \infty)$ satisfying the following (i) (iii):

(i) We have $F_{0}(r)=F(r)$ and $V_{0}(r)=V(r)$ for almost all $r \in[0, \infty)$.

(ii) $F_{n}(r)$ converges to $F_{0}(r)$ in $H$ uniformly on every finite interval in $[0, \infty)$. Similarly $F_{n}^{\prime}(r)$ converges to $V_{0}(r)$ in $H$ uniformly on every finite interval in $[0, \infty)$, and hence $F_{0}(r)$ and $V_{0}(r)$ are strongly continuous on $[0, \infty)$.

(iii) We have for any $u \in H$ and any $r, c \in[0, \infty)$

$$
\left(F_{0}(r), u\right)_{H}=\left(F_{0}(c), u\right)_{H}+\int_{c}^{r}\left(V_{0}(t), u\right)_{H} d t
$$

and

$$
\left(V_{0}(r), u\right)_{H}=\left(V_{0}(c), u\right)_{H}+\int_{c}^{r}(Y(t), u)_{H} d t,
$$

where $Y(r)=\lim F_{n}^{\prime \prime}(r)$ in $L_{1}[0, \beta ; H]$ for any $\beta \geqq 0$.

Proof. Let us define $F_{0}(r)$ and $V_{0}(r)$ by

$$
\begin{cases}F_{0}(r)=\lim _{n \rightarrow \infty} F_{n}(r) & \text { in } H, \\ V_{0}(r)=\lim _{n \rightarrow \infty} F_{n}^{\prime}(r) & \text { in } H .\end{cases}
$$

By Lemma 3.6 and Lemma 3.7, the sequences $F_{n}(r)$ and $F_{n}^{\prime}(r)$ converge uniformly on every finite interval in $[0, \infty)$, and hence $F_{0}(r)$ and $V_{0}(r)$ are strongly continuous. On the other hand we have

$$
F=\lim F_{n} \quad \text { in } \mathfrak{h},
$$


and in view of Lemma 3.5 we have

$$
V=\lim F_{n}^{\prime} \quad \text { in } \quad L_{2}(0, \beta ; H)
$$

for any $\beta \geqq 0$. Therefore we have $F_{0}(r)=F(r)$ and $V_{0}(r)=V(r)$ for almost all $r \geqq 0$. Letting $n \rightarrow \infty$ in the relation

$$
\left(F_{n}(r), u\right)_{H}=\left(F_{n}(c), u\right)_{H}+\int_{c}^{r}\left(F_{n}^{\prime}(t), u\right)_{H} d t
$$

we obtain (3.26). Similarly we obtain (3.27), noting that the existence of $Y(t)$ was proved in Lemma 3.7.

Q.E. D.

Proof of Theorem 3.2. Let $F_{0}(r)$ be as in Lemma 3.8. Since $F_{0}(r)$ $=F(r)$ for almost all $r \in\left[0, \infty\right.$ ) by (i) of Lemma 3.8. and $\lim _{n \rightarrow \infty} F_{n}=F$ in $\mathfrak{h}$, we have $F_{n} \rightarrow F_{0}$ in $\mathfrak{h}$. It follows from (3.25) that $F_{0}(r)$ is strongly continuously differentiable with its strong derivative $F_{0}^{\prime}(r)=V_{0}(r)$. Similarly (3.26) implies that $V_{0}(r)$ is weakly absolutely continuous and is weakly differentiable almost everywhere with its weak derivative $V_{0}^{\prime}(r)=Y(r)$. Therefore $F_{0}(r)$ satisfies (i) of Definition 3.1. We show that $F_{0}(r)$ satisfies the boundary condition (3.1). Since we have $F_{n}(0) \rightarrow F_{0}(0)$ and $F_{n}^{\prime}(0)$ $\rightarrow F_{0}^{\prime}(0)=V_{0}(0)$ by Lemma 3.8 , we obtain

$$
B F_{0}^{\prime}(0)-C F_{0}^{\prime}(0)=\lim _{n \rightarrow \infty}\left(B F_{n}^{\prime}(0)-C F_{n}(0)\right)=\lim _{n \rightarrow \infty} 0=0 .
$$

Thus we have shown that $F_{0}$ satisfies (ii) of Definition 3.1.

Next we show that $\mathscr{L} F_{0}=W$ in $\mathfrak{h}$, where $W=\lim _{n \rightarrow \infty} W_{n}=\lim _{n \rightarrow \infty} L F_{n}$. We defined in Lemma 3.8

$$
V_{0}^{\prime}(r)=Y(r)=\lim _{n \rightarrow \infty} F_{n}^{\prime \prime}(r) \quad \text { in } L_{1}(0, \beta ; H)
$$

for any $\beta \geqq 0$. As $F_{n}^{\prime \prime}(r)=A(r) F_{n}(r)-W_{n}(r)$, we have

$$
Y(r)=\lim _{n \rightarrow \infty}\left(A(r) F_{n}(r)-W_{n}(r)\right) \quad \text { in } L_{1}(0, \beta ; H) .
$$

On the other hand $\lim _{n \rightarrow \infty} W_{n}=W$ in $\mathfrak{h}$ and we have for $\beta \geqq 0$

$$
\begin{aligned}
& \int_{0}^{\beta}\left\|A(r) F_{n}(r)-A(r) F_{0}(r)\right\|_{H} d r \\
& \quad \leqq \int_{0}^{\beta}\|A(r)\| d r \cdot \max _{0 \leqq r \leqq \beta}\left\|F_{n}(r)-F_{0}(r)\right\|_{H \rightarrow 0},
\end{aligned}
$$

which implies $A(r) F_{n}(r) \rightarrow A(r) F_{0}(r)$ in $L_{1}(0, \beta ; H)$. Here we have made use of the uniform convergence of $F_{n}(r)$ on $[0, \beta]$. It follows from 
(3.33) and (3.32) that

$$
Y(r)=A(r) F_{0}(r)-W(r) \quad \text { in } L_{1}(0, \beta ; H),
$$

and hence we have for almost all $r \in[0, \infty)$

$$
\begin{aligned}
W(r)=-Y(r)+A(r) F_{0}(r) & =-V_{0}^{\prime}(r)+A(r) F_{0}(r) \\
& =-F_{0}^{\prime \prime}(r)+A(r) F_{0}(r),
\end{aligned}
$$

which implies that $\mathscr{L} F_{0}=W$. Thus we have shown that $F_{0} \in \mathscr{D}$ and $L F_{0}$ $=W$. Since $F=F_{0}$ in $\mathfrak{h}$, the closedness of $L$ has been completely proved. (3.4) and (3.5) are obtained immediately from ii) of Lemma 3.8, which completes the proof.

Q.E. D.

\section{§4. The Eigenfunction Expansion}

Let $L$ be the closed operator discussed in the preceding section. Now we shall prove the eigenfunction expansion for self-adjoint operators which will be obtained as a restriction of $L$.

Now let us assume that there exists a self-adjoint restriction of $L$, which will be denoted by $L_{0}$, that is, $L_{0}$ is a self-adjoint operator and $L_{0} \leqq L{ }^{7}{ }^{7}$ We denote by $E(\mu)$ the resolution of the identity associated with $L_{0} . E(\mu)$ is assumed to be right continuous.

First we give the definition of a sort of Riemann-Stieltjes integral which will be useful in the section.

Definition 4.1. (1) Let $\left[\mu_{1}, \mu_{2}\right]$ be a finite, closed interval in $\mathbf{R}=$ $(-\infty, \infty)$. Let $Q(\mu)$ and $\alpha(\mu)$ be B-valued functions on $\left[\mu_{1}, \mu_{2}\right]$ and let $U(\mu)$ be an $H$-valued function on $\left[\mu_{1}, \mu_{2}\right]$. Denote by $\Delta$ a finite subdivision of the interval $\left[\mu_{1}, \mu_{2}\right]$ determined by the points $\eta_{0}, \eta_{1}, \ldots, \eta_{n}$, where

$$
\mu_{1}=\eta_{0}<\eta_{1}<\cdots<\eta_{n}=\mu_{2}
$$

7) Let, for example, $\|A(r)\|$ be bounded on $[0, \infty)$. Then defining $L_{1} F=\mathscr{L} F$ for every function $F(r)$ which satisfies (i) and (ii) of Definition 3.1 and has a compact carrier, we can show that $L_{1}$ is a semi-bounded, symmetric operator. The Friedrichs extention of $L_{1}$ becomes a self-adjoint restriction of $L$. 
By the norm $\delta=\delta(\Delta)$ of $\Delta$ we mean the largest of the numbers $\eta_{i+1}-\eta_{i}$ $(i=0,1,2, \ldots, n-1)$.

If the limit

$$
\lim _{\delta(\Delta) \rightarrow 0} \sum_{i=0}^{n-1} Q\left(\eta_{i}^{\prime}\right)\left(\alpha\left(\eta_{i_{+} 1}\right)-\alpha\left(\eta_{i}\right)\right) U\left(\eta_{i}^{\prime}\right)
$$

or

$$
\lim _{\delta(\Delta) \rightarrow 0} \sum Q\left(\eta_{i}^{\prime}\right)\left(U\left(\eta_{i+1}\right)-U\left(\eta_{i}\right)\right)
$$

where $\eta_{i} \leqq \eta_{i}^{\prime} \leqq \eta_{i+1}(i=0,1,2, \ldots, n-1)$, exists in the sense of weak convergence in $H$ independently of the manner of subdivision and of the choice of the numbers $\eta_{i}^{\prime}$, then the limit is denoted by $\int_{\left[\mu_{1}, \mu_{2}\right]} Q(\mu) \alpha(d \mu) U(\mu)$ or $\int_{\left[\mu_{1}, \mu_{2}\right]} Q(\mu) U(d \mu)$.

(2) Suppose that for any $\mu_{\in}\left(\mu_{1}, \mu_{2}\right]$ the integral

$$
\int_{\left[\mu, \mu_{2}\right]} Q(\eta) \alpha(d \eta) U(\eta) \text { or } \int_{\left[\mu, \mu_{2}\right]} Q(\eta) U(d \eta)
$$

exists in the sense of (1) and suppose that there exists

$$
\lim _{\mu \rightarrow \mu_{1}+0} \int_{\left[\mu, \mu_{2}\right]} Q(\eta) \alpha(d \eta) U(\eta) \quad \text { or } \lim _{\mu \rightarrow \mu_{1}+0} \int_{\left[\mu, \mu_{2}\right]} Q(\eta) U(d \eta)
$$

in the sense of weak convergence in $H$. Then we define the integral on the half-open interval $\left(\mu_{1}, \mu_{2}\right]$ by

$$
\int_{\left(\mu_{1}, \mu_{2}\right]} Q(\eta) \alpha(d \eta) U(\eta)=\lim _{\mu \rightarrow \mu_{1}+0} \int_{\left[\mu, \mu_{2}\right]} Q(\eta) \alpha(d \eta) U(\eta)
$$

or

$$
\int_{\left(\mu_{1}, \mu_{2}\right]} Q(\eta) U(d \eta)=\lim _{\mu \rightarrow \mu_{1}+0} \int_{\left[\mu, \mu_{2}\right]} Q(\eta) U(d \eta) .
$$

We state two lemmas which give sufficient conditions for the existence of the integrals defined above. We shall give the proof of these lemmas in the Appendix.

Lemma 4.2. Denote by $\left[\mu_{1}, \mu_{2}\right]$ a finite, closed interval in $\mathbf{R}$. Let $Q(\mu)$ be a B-valued function on $\left[\mu_{1}, \mu_{2}\right]$ which is continuously differentiable in the uniform topology of $\mathbf{B}$ with its derivative $\frac{d Q}{d \mu} \in \mathbf{B}$. Let $U(\mu)$ be an $H$-valued, strongly continuously differentiable function on $\left[\mu_{1}, \mu_{2}\right]$ with its strong derivative $\frac{d U}{d \mu}$. Let $\alpha(\mu)$ be a $\mathbf{B}$-valued function on $\left[\mu_{1}, \mu_{2}\right]$ 
such that $\|\alpha(\mu)\|$ is bounded on $\left[\mu_{1}, \mu_{2}\right]$ and $\left(\frac{d Q}{d \mu} \alpha(\mu) U(\mu)+Q(\mu) \alpha(\mu)\right.$ $\left.\frac{d U}{d \mu}, u\right)_{H}$ is integrable on $\left[\mu_{1}, \mu_{2}\right]$ in the sense of Riemann for $u \in H$. Then the integral $\int_{\left[\mu_{1}, \mu_{2}\right]} Q(\mu) \alpha(d \mu) U(\mu)$ exists in the sense of Definition 4.1 and we have for any $u \in H$

$$
\begin{aligned}
& \left(\int_{\left[\mu_{1}, \mu_{2}\right]} Q(\mu) \alpha(d \mu) U(\mu), u\right)_{H} \\
& \quad=\left(Q\left(\mu_{2}\right) \alpha\left(\mu_{2}\right) U\left(\mu_{2}\right), u\right)_{H}-\left(Q\left(\mu_{1}\right) \alpha\left(\mu_{1}\right) U\left(\mu_{1}\right), u\right)_{H} \\
& \quad-\int_{\mu_{1}}^{\mu_{2}}\left(\frac{d Q}{d \mu} \alpha(\mu) U(\mu)+Q(\mu) \alpha(\mu) \frac{d U}{d \mu}, u\right)_{H} d \mu .
\end{aligned}
$$

Lemma 4.3. Denote by $\left[\mu_{1}, \mu_{2}\right]$ a finite closed interval in $\mathbf{R}$. Let $Q(\mu)$ as in Lemma 4.3. Let $V(\mu)$ be an H-valued function on $\left[\mu_{1}, \mu_{2}\right]$ such that $\|V(\mu)\|_{H}$ is bounded on $\left[\mu_{1}, \mu_{2}\right]$ and $\left(\frac{d Q}{d \mu}-V(\mu), u\right)_{H}$ is integrable on $\left[\mu_{1}, \mu_{2}\right]$ in the sense of Riemann for any $u \in H$. Then the integral $\int_{\left[\mu_{1}, \mu_{2}\right]} Q(\mu) V(d \mu)$ exists in the sense of Definition 4.1 and we have for any $u \in H$

$$
\begin{aligned}
\left(\int_{\left[\mu_{1}, \mu_{2}\right]} Q(\mu) V(d \mu), u\right)_{H}= & \left(Q\left(\mu_{2}\right) V\left(\mu_{2}\right), u\right)_{H}-\left(Q\left(\mu_{1}\right) V\left(\mu_{1}\right), u\right) \\
& -\int_{\mu_{1}}^{\mu_{2}}\left(\frac{d Q}{d \mu} V(\mu), u\right)_{H} d \mu .
\end{aligned}
$$

We shall show how the resolution of the identity $E(\mu)$ of $L_{0}$ and the eigenoperator $\phi(r, \lambda)$ are related each other.

Proposition 4.4.8) Let $F \in \mathfrak{h}$ and let $I=\left(\mu_{1}, \mu_{2}\right]$ be a finite halfopen interval in $\mathbf{R}$. Then we have

$$
F(r, I)=\int_{I} \phi(r, \mu)\left(P_{1} F^{\prime}(0, d \mu)+B_{2}^{-1} P_{2} F(0, d \mu)\right),
$$

where $P_{i}(i=1,2)$ are projection operators onto $H_{i}(i=1,2)$ defined in Definition 1.8 and

8) Cf. Kodaira [4], p. 188, (4.12); or [5], p. 930, (2.6). 


$$
\left\{\begin{array}{l}
F(r, I)=(E(I) F)(r)=\left(E\left(\mu_{2}\right)-E\left(\mu_{1}\right)\right) F(r), \\
F(r, \mu)=(E(\mu)-E(0)) F(r), \\
F^{\prime}(r, \mu)=((E(\mu)-E(0)) F)^{\prime}(r) .
\end{array}\right.
$$

The integral of the right-hand side of (4.10) exists in the sense of Definition 4.1.

To prove this proposition we prepare some lemmas. We have to show that the integral of the right-hand side of (4.10) exists.

Lemma 4.5. Let $F \in \mathfrak{h}$ and let $F(r, \mu)$ and $F^{\prime}(r, \mu)$ be as in Proposition 4.2 .

(i) Then for an arbitrarily fixed $r \in[0, \infty), F(r, \mu)$ and $F^{\prime}(r, \mu)$, as functions of $\mu \in \mathbf{R}$, are strongly right continuous on $\mathbf{R}$ and the strong limits

$$
\begin{cases}\substack{\mathrm{s}-\lim _{\eta \rightarrow \mu-0} \\ \mathrm{~s}-\lim _{\eta \rightarrow \mu-0}} & F(r, \eta) \\ F^{\prime}(r, \eta)\end{cases}
$$

exist for any $\mu \in \mathbf{R}$. Therefore for each $r \in[0, \infty)\|F(r, \mu)\|$ and $\left\|F^{\prime}(r, \mu)\right\|$ are bounded functions on every finite, closed interval $\left[\mu_{1}, \mu_{2}\right] \subseteq \mathbf{R}$.

(ii) Denote by $d_{0}(r)$ (or $d_{1}(r)$ ) the set of all discontinuous points of $F(r, \mu)\left(\right.$ or $\left.F^{\prime}(r, \mu)\right)$ and denote by $d=d\left(L_{0}\right)$ the set of all discontinuous points of $E(\mu)$. Then we have for any $r \in[0, \infty)$

$$
d_{i}(r) \subseteq d \quad(i=0,1)
$$

and hence both $d_{0}(r)$ and $d_{1}(r)$ are enumerable sets.

(iii) For $F \in \mathfrak{h}$ the integral of the right-hand side of (4.10) exists in the sense of Definition 4.1 and we have for $u \in H$

$$
\begin{aligned}
& \left(\int_{\left(\mu_{1}, \mu_{2}\right]} \phi(r, \mu)\left(P_{1} F^{\prime}(0, d \mu)+B_{2}^{-1} P_{2} F(0, d \mu)\right), u\right)_{H} \\
& =\left.\phi(r, \mu)\left(P_{1} F^{\prime}(0, \mu)+B_{2}^{-1} P_{2} F(0, \mu), u\right)_{H}\right|_{\mu=\mu_{1}} ^{\mu=\mu_{2}} \\
& \quad-\int_{\mu_{1}}^{\mu_{2}}\left(\frac{\partial \phi(r, \mu)}{\partial \mu}\left(P_{1} F^{\prime}(0, \mu)+B_{2}^{-1} P_{2} F(0, \mu)\right), u\right)_{H} d \mu .
\end{aligned}
$$


Here the integral of the right-hand side of (4.14) exists in the sense of Riemann integral on $\left[\mu_{1}, \mu_{2}\right]$.

Proof. Putting $\mathrm{s}_{\eta \rightarrow \mu-0} F(r, \eta)=F(r, \mu-0)$, we show that $F(r, \mu-0)$ exists for any $r \geqq 0$ and $\mu \in \mathbf{R}$ and if $E(\mu)=E(\mu-0)$ then $F(r, \mu-0)=$ $F(r, \mu)$. In fact take a sequence $\mu_{n}$ such that $\mu_{n}<\mu$ and $\mu_{n} \rightarrow \mu_{\text {. Then }}$ we have

$$
\begin{cases}\left(E\left(\mu_{n}\right)-E(\mu)\right) F \rightarrow(E(\mu-0)-E(\mu)) F & \text { in } \mathfrak{h}, \\ L\left(E\left(\mu_{n}\right)-E(\mu)\right) F \rightarrow L(E(\mu-0)-E(\mu)) F & \text { in } \mathfrak{h} .\end{cases}
$$

Here we have made use of the fact that $L_{0} \subseteq L$. Then we can apply Theorem 3.2 to obtain

$$
F\left(r, \mu_{n}\right)=\left(E\left(\mu_{n}\right)-E(0)\right) F(r) \rightarrow(E(\mu-0)-E(0)) F(r) \text { in } H .
$$

Thus we have shown that $\operatorname{s-lim}_{\eta \rightarrow \mu-0} F(r, \eta)$ exists and

$$
F(r, \mu-0)=(E(\mu-0)-E(0)) F(r)
$$

holds for $r \geqq 0$ and $\mu \in \mathbf{R}$. If $E(\mu-0)=E(\mu)$, then we have

$$
F(r, \mu-0)=(E(\mu)-E(0)) F(r)=F(r, \mu) .
$$

Similarly we can show $F(r, \mu)$ is strongly right continuous. In the same way we can prove that $F^{\prime}(r, \mu)$ is strongly right continuous and that $\mathrm{s}-\lim _{\rightarrow \rightarrow-0} F^{\prime}(r, \eta)=F^{\prime}(r, \mu-0)$ exists and $F^{\prime}(r, \mu-0)=F^{\prime}(r, \mu)$ if $E(\mu)=$ $\stackrel{\rightarrow \rightarrow \mu-0}{E(\mu-0)}$. Thus (i) and (ii) of Lemma 4.5 has been proved.

Let $r \in[0, \infty)$ be fixed. Then putting

$$
\left\{\begin{array}{l}
Q(\mu)=\phi(r, \mu), \\
V(\mu)=P_{1} F^{\prime}(0, \mu)+B_{2}^{-1} P_{2} F(0, \mu),
\end{array}\right.
$$

we can show that $Q(\mu)$ and $V(\mu)$ satisfy the condition of Lemma 4.3 , and hence there exists the integral $\int_{\left(\mu_{1}, \mu_{2}\right]} Q(\mu) V(d \mu)$. In fact it follows from Proposition 1.6 and Proposition 1.9 that $\phi(r, \lambda)$ and $\frac{\partial \phi(r, \lambda)}{\partial \lambda}$, as functions of $\mu$, are continuous in the uniform topology of $\mathbf{B}$. By (i) $\|V(\mu)\|_{H}$ is bounded on $\left[\mu_{1}, \mu_{2}\right]$. Further, (i) and (ii) imply that $\left(\frac{\partial \phi(r, \mu)}{\partial \mu} V(\mu), u\right)_{H}$ 
is at most discontinuous of the first kind for any $u \in H$ and that the set of all discontinuous points of $\left(\frac{\partial \phi(r, \mu)}{\partial} \mu V(\mu), u\right)_{H}$ is an enumerable set. Hence $\left(\frac{\partial \phi(r, \mu)}{\partial \mu} V(\mu), u\right)_{H}$ is integrable on $\left[\mu_{1}, \mu_{2}\right]$ in the sense of Riemann.

Therefore by Lemma 4.3 there exists the integral

$$
\int_{\left[\mu, \mu_{2}\right]} Q(\eta) V(d \eta)
$$

for $\mu \in\left(\mu_{1}, \mu_{2}\right]$ and we have for $u \in H$

$$
\begin{aligned}
\left(\int_{\left[\mu_{,} \mu_{2}\right]} Q(\eta) V(d \eta), u\right)_{H}= & \left.(Q(\eta), V(\eta), u)_{H}\right|_{\eta=\mu} ^{\eta=\mu_{2}} \\
& -\int_{\mu}^{\mu_{2}}\left(\frac{\partial Q(\eta)}{\partial \eta} V(\eta) u\right)_{H} d \eta .
\end{aligned}
$$

Since $V(\eta)$ is strongly right continuous, there exists

$$
\begin{aligned}
\lim _{\mu \rightarrow \mu_{1}+0}\left(\int_{\left[\mu, \mu_{2}\right]} Q(\eta) V(d \eta, u)_{H}=\right. & \left.(Q(\eta) V(\eta), u)_{H}\right|_{\eta=\mu_{1}} ^{\eta=\mu_{2}} \\
& -\int_{\mu_{1}}^{\mu_{2}}\left(\frac{\partial Q(\eta)}{\partial \eta} V(\eta), u\right)_{H} d \eta
\end{aligned}
$$

which completes the proof.

Q. E. D.

Put

$$
U(r, \mu)= \begin{cases}\int_{(0, \mu]} \phi(r, \eta) V(d \eta) & \text { if } \mu>0, \\ 0 & \text { if } \mu=0, \\ -\int_{(\mu, 0]} \phi(r, \eta) V(d \eta) & \text { if } \mu<0,\end{cases}
$$

where

$$
V(\mu)=P_{1} F^{\prime}(0, \mu)+B_{2}^{-1} P_{2} F(0, \mu) .
$$

We shall show some properties of $U(r, \mu)$.

Lemma 4.6. Let $r \in[0, \infty)$ and $\mu \in \mathbf{R}$.

(i) Then for any $\mu \in \mathbf{R} U(\cdot, \mu) \in \mathbf{D}$. And $\|U(r, \mu)\|_{H}$ is locally bounded on $[0, \infty) \times \mathbf{R}$.

(ii) There exists the integral $\int_{\left(\mu_{1}, \mu_{2}\right]} \eta U(r, d \eta)$ on every finite halfopen interval $\left(\mu_{1}, \mu_{2}\right]$ and for every $r \in[0, \infty)$. Putting 


$$
V(r, \mu)= \begin{cases}\int_{(0, \mu]} \eta U(r, d \eta) & \text { if } \mu>0, \\ 0 & \text { if } \mu=0, \\ -\int_{(\mu, 0]} \eta U(r, d \eta) & \text { if } \mu<0,\end{cases}
$$

we have a null set $\boldsymbol{e}$ in $[0, \infty)$ such that for $r \in[0, \infty)-e$

$$
\mathscr{L} U(r, \mu)=V(r, u)
$$

holds, where we define $\mathscr{L} U(r, \mu)$ by $-U^{\prime \prime}(r, \mu)+A(r) U(r, \mu)$.

(iii) We have for any $\mu \in \mathbf{R}$

$$
\left\{\begin{array}{l}
U(0, \mu)=F(0, \mu), \\
U^{\prime}(0, \mu)=F^{\prime}(0, \mu) .
\end{array}\right.
$$

Proof. By the formula (4.14) and Proposition 1.6 and Proposition 1.9 it is easy to see that for each $\mu \in \mathbf{R} \quad U(r, \mu) \in \mathbf{D}$ and $\|U(r, \mu)\|_{H}$ is locally bounded on $[0, \infty) \times \mathbf{R}$. If we put

$$
\tilde{V}(r, \mu)= \begin{cases}\int_{(0, \mu]} \eta \phi(r, \eta) V(d \eta) & \text { if } \mu>0, \\ 0 & \text { if } \mu=0, \\ -\int_{(\mu, 0]} \eta \phi(r, \eta) V(d \eta) & \text { if } \eta<0,\end{cases}
$$

then it follows from Proposition 1.6 and Proposition 1.9 that we have for almost all $r \in[0, \infty)$

$$
\mathscr{L} U(r, \mu)=\tilde{V}(r, \mu) .
$$

Again using (4.14), we can see that for each $r \in[0, \infty)\|U(r, \mu)\|_{H}$ is locally bounded on $\mathbf{R}$ and $(U(r, \mu), u)_{H}$ is integrable on any finite interval in $\mathbf{R}$ in the sense of Riemann, where $u$ is an arbitrary element of $H$. Moreover $U(r, \mu)$, as a function of $\mu$, is right-continuous. Therefore by Lemma 4.3 the integral $\int_{\left(\mu_{1}, \mu_{2}\right]} \eta U(r, d \eta)$ exists and $V(r, \mu)$ is well-defined.

We shall show $V(r, \mu)=\tilde{V}(r, \mu)$ on $[0, \infty) \times \mathbf{R}$. It follows from (4.25) and the right continuity of $U(r, \mu)$ that

$$
\begin{aligned}
(V(r, \mu), u) & =\left.(\eta U(r, \eta), u)\right|_{\eta=0} ^{\eta=\mu}-\int_{0}^{\mu}(U(r, \eta), u) d \eta \\
& =\mu(U(r, \mu), u)-\int_{0}^{\mu}(U(r, \eta), u) d \eta
\end{aligned}
$$


holds for $u \in H$. Noting (4.23) we obtain

$$
\begin{gathered}
\mu(U(r, \mu), u)=\mu(\phi(r, \mu) V(\mu), u)-\mu(\phi(r, 0) V(\mu), u) \\
-\mu \int_{0}^{\mu}\left(\frac{\partial \phi(r, \eta)}{\partial \eta} V(\eta), u\right) d \eta
\end{gathered}
$$

and

$$
\begin{aligned}
\int_{0}^{\mu}(U(r, \eta), u) d \eta= & \int_{0}^{\mu}\{(\phi(r, \eta) V(\eta), u)-(\phi(r, 0) V(0), u)\} d \eta \\
& -\int_{0}^{\mu} \int_{0}^{\eta}\left(\frac{\partial \phi(r, \lambda)}{\partial \lambda} V(\lambda), u\right) d \lambda d \eta \\
= & \int_{0}^{\mu}(\phi(r, \eta) V(\eta), u) d \eta-\mu(\phi(r, 0) V(0), u) \\
& -\int_{0}^{\mu}(\mu-\lambda)\left(\frac{\partial \phi(r, \lambda)}{\partial \lambda} V(\lambda), u\right) d \lambda
\end{aligned}
$$

By (4.30), (4.31) and (4.32) we have for $u \in H$

(4.33) $\quad(V(r, \mu), u)=\mu(\phi(r, \mu) V(\mu), u)-\int_{0}^{\mu}\left(\frac{\partial}{\partial \eta}(\eta \phi(r, \eta)) V(\eta), u\right) d \eta$.

On the other hand from (4.28) we obtain

$$
(\tilde{V}(r, \mu), u)=\mu(\phi(r, \mu) V(\mu), u)-\int_{0}^{\mu}\left(\frac{\partial}{\partial \eta}(\eta \phi(r, \eta)) V(\eta), u\right) d \eta
$$

and hence $V(r, \mu)=\tilde{V}(r, \mu)$.

Let us show (4.27). For any $u \in H$

$$
\begin{gathered}
(U(0, \mu), u)=(\phi(0, \mu) V(\mu), u)-(\phi(0,0) V(0), u) \\
-\int_{0}^{\mu}\left(\frac{\partial \phi(0, \eta)}{\partial \eta} V(\eta), u\right) d \eta
\end{gathered}
$$

holds. Recalling Definition 1.8 we have for any $\mu \in \mathbf{R}$

$$
\phi(0, \mu)=B \text {. }
$$

It follows from (4.35), (4.36) and (4.24)

$$
\begin{aligned}
(U(0, \mu), u) & =(B(V(\mu)-V(0)), u)=(B V(\mu), u) \\
& =\left(B\left(P_{1} F^{\prime}(0, \mu)+B_{2}^{-1} P_{2} F(0, \mu)\right), u\right) .
\end{aligned}
$$

From Definition 1.8 we have $B P_{1}=0$ and $B B_{2}^{-1} P_{2}=P_{2}$. As $(E(\mu)-E(0)) F$ 
$\in \mathscr{D}$, we have $P_{1} F(0, \mu)=0$. Therefore $(4.37)$ becomes

$$
(U(0, \mu), u)=(F(0, \mu), u),
$$

and hence $U(0, \mu)=F(0, \mu)$. Similarly, noting that

$$
U^{\prime}(r, \mu)= \begin{cases}\int_{(0, \mu]} \phi^{\prime}(r, \eta) V(d \eta) & \text { if } \mu>0, \\ 0 & \text { if } \mu=0, \\ -\int_{(\mu, 0]} \phi^{\prime}(r, \eta) V(d \eta) & \text { if } \eta<0,\end{cases}
$$

we obtain $U^{\prime}(0, \mu)=F^{\prime}(0, \mu)$, which completes the proof.

Q.E. D.

In the following lemma we shall show that $F(r, \mu)$ satisfies the integral equation (4.26) as well as $U(r, \mu)$.

Lemma 4.7 Let $F \in \mathfrak{h}$ and let $F(r, \mu)$ be as in (4.11).

(i) Then for any $\mu \in \mathbf{R}$ there exists a null set $e(\mu)$ in $[0, \infty)$ such that

$$
\text { (4.40) } \mathscr{L} F(r, \mu)= \begin{cases}\int_{(0, \mu]} \eta F(r, d \eta) & \text { if } \mu>0, \\ 0 & \text { if } \mu=0, \\ -\int_{(0, \mu]} \eta F(r, d \eta) & \text { if } \mu<0\end{cases}
$$

holds for all $r \in[0, \infty)-e(\mu)$. Here the integral of the right-hand side of (4.40) exists in the sense of Definition 4.1.

(ii) $\|F(r, \mu)\|_{H}$ is locally bounded on $[0, \infty) \times \mathbf{R}$.

Proof. We shall prove (4.40) assuming $\mu>0$. Using (i) and (ii) of Lemma 4.5 we can easily show that the integral $\int_{(0, \mu]} \eta d F(r, \eta)$ exists. Since $F(r, \mu)=(E(\mu)-E(0)) F(r)$, by spectral representation of $L_{0}$ we have for $G \in \mathfrak{h}$

$$
\begin{aligned}
\left(L_{0} F(\cdot, \mu), G(\cdot)\right)_{\mathfrak{h}} & =\int_{(0, \mu]} \eta d(F(\cdot, \eta), G(\cdot))_{\mathfrak{h}} \\
& =\mu(F(\cdot, \eta), G(\cdot))_{\mathfrak{h}}-\int_{0}^{\mu}(F(\cdot, \eta), G(\cdot))_{\mathfrak{h}} d \eta .
\end{aligned}
$$

Notice that $L_{0} \subseteq L$. Then from (4.41) we obtain 


$$
\begin{aligned}
& \int_{0}^{\infty}(\mathscr{L} F(r, \mu), G(r))_{H} d r=\int_{0}^{\infty} \mu(F(r, \mu), G(r))_{H} d r \\
&-\int_{0}^{\mu} \int_{0}^{\infty}(F(r, \eta), G(r))_{H} d r d \eta .
\end{aligned}
$$

Using Schwartz' inequality, we can estimate the integral in the right-hand side of (4.42) to obtain

$$
\begin{aligned}
\int_{0}^{\mu} \int_{0}^{\infty}\left|(F(r, \eta), G(r))_{H}\right| d r d \eta & \leqq \int_{0}^{\mu} \int_{0}^{\infty}\|F(r, \eta)\|_{H}|| G(r) \|_{H} d r d \eta \\
& \leqq \int_{0}^{\mu}\|(E(\eta)-E(0)) F\|_{\mathfrak{h}}\left\|_{i} G\right\|_{\mathfrak{h}} d \eta \\
& \leqq \mu\|F\|_{\mathfrak{h}}\|G\|_{\mathfrak{h}} .
\end{aligned}
$$

Hence by Fubini's theorem we have

$$
\begin{aligned}
\int_{0}^{\infty}(\mathscr{L} F(r, \mu), G(r)) d r & =\int_{0}^{\infty}\{\mu(F(r, \mu), G(r)) \\
& \left.\quad-\int_{0}^{\infty}(F(r, \eta), G(r)) d \eta\right\} d r \\
= & \int_{0}^{\infty}\left(\int_{(0, \mu]} \eta F(r, d \eta), G(r)\right)_{H} d r,
\end{aligned}
$$

where, noting (4.43), we can see that $\int_{(0, \mu]} \eta F(r, d \eta) \in \mathfrak{h}$. Thus it follows from (4.44) that $\left(\mathscr{L} F(\cdot, \mu)-\int_{(0, \mu]} \eta F(\cdot, d \eta), G\right)_{\mathfrak{h}}=0$ holds for all $G \in \mathfrak{h}$ and hence $\mathscr{L} F(r, \mu)=\int_{(0, \mu]} \eta F(r, d \eta)$ for almost all $r$. Quite similarly we can prove the case $\mu<0$. If $\mu=0$, then clearly both sides of (4.40) are equal to zero, for $F(r, 0) \equiv 0$ on $[0, \infty)$.

Next we shall prove (ii). We take $\beta>0$ and a finite, closed interval $\left[\mu_{1}, \mu_{2}\right]$ in $\mathbf{R}$. Further we take a smooth function $p(r)$ on $[0, \infty)$ such that $0 \leqq p(r) \leqq 1, p(r)=1$ on $[0, \beta]$, and $p(r)=0$ on $[\beta+1, \infty)$. Replacing $F(r)$ and $W(r)$ by $F(r, \mu)-F\left(r, \mu_{1}\right)$ and $L\left(E(\mu)-E\left(\mu_{1}\right)\right) F(r)$ respectively in (3.8) in Lemma 3.4, we have

$$
\begin{aligned}
&\left\|p\left(F^{\prime}(\cdot, \mu)-F^{\prime}\left(\cdot, \mu_{1}\right)\right)\right\|_{\mathfrak{h}}^{2} \\
&=\left(p L\left(E(\mu)-E\left(\mu_{1}\right)\right) F, F(\cdot, \mu)-F\left(\cdot, \mu_{1}\right)\right)_{\mathfrak{G}} \\
&-2\left(p\left(F^{\prime}(\cdot, \mu)-F^{\prime}\left(\cdot, \mu_{1}\right)\right), p^{\prime}\left(F(\cdot, \mu)-F\left(\cdot, \mu_{1}\right)\right)\right)_{\mathfrak{h}} \\
&-\left(A p\left(F(\cdot, \mu)-F\left(\cdot, \mu_{1}\right)\right), p\left(F(\cdot, \mu)-F\left(\cdot, \mu_{1}\right)\right)\right)_{\mathfrak{h}}
\end{aligned}
$$




$$
\begin{aligned}
& -\left(B_{2}^{-1} C_{2} P_{2}\left(F(0, \mu)-F\left(0, \mu_{1}\right)\right), F(0, \mu)-F\left(0, \mu_{1}\right)\right)_{H} \\
= & K_{1}+K_{2}+K_{3}+K_{4} .
\end{aligned}
$$

Let $\mu \in\left[\mu_{1}, \mu_{2}\right]$, then $K_{i}(i=1,2,3,4)$ are estimated as follows:

$$
\begin{gathered}
\left|K_{1}\right| \leqq\left\|L\left(E(\mu)-E\left(\mu_{1}\right)\right) F\right\|_{\mathfrak{h}}\left\|\left(E(\mu)-E\left(\mu_{1}\right)\right) F\right\|_{\mathfrak{h}} \\
\leqq \max \left(\left|\mu_{1}\right|,\left|\mu_{2}\right|\right) \cdot\|F\|_{\mathfrak{h} \cdot}^{2} \\
\left|K_{2}\right| \leqq 2\left\|p\left(F^{\prime}(\cdot, \mu)-F^{\prime}\left(\cdot, \mu_{1}\right)\right)\right\|_{\mathfrak{h}} \cdot C(p)\|F\|_{\mathfrak{h}},
\end{gathered}
$$

where $C(p)$ is a positive constant depending only on $p(r)$.

$$
\left|K_{3}\right| \leqq\left. C_{\beta+1}|| F\right|_{h} ^{2}
$$

where we have made use of (3) of Assumption 1.1.

$$
\left|K_{4}\right| \leqq\left\|B_{2}^{-1}||\right\| C_{2}\|\| F(0, \mu)-F\left(0, \mu_{1}\right) \|_{H}^{2} .
$$

Notice that $\left\|F\left(0, \mu_{0}\right)\right\|$ is bounded on $\left[\mu_{1}, \mu_{2}\right]$ by (i) of Lemma 4.5. Then we can see that there exists a constant $K=K\left(\mu_{1}, \mu_{2}, \beta, F\right)$ such that

$$
\int_{0}^{\beta}\left\|F^{\prime}(r, \mu)-F^{\prime}\left(r, \mu_{1}\right)\right\|_{H}^{2} d r \leqq K\left(\mu_{1}, \mu_{2}, \beta, F\right)
$$

holds for $\mu \in\left[\mu_{1}, \mu_{2}\right]$, which implies that $\int_{0}^{\beta}\left\|F^{\prime}(r, \mu)\right\|_{H}^{2} d r$ is bounded on $\left[\mu_{1}, \mu_{2}\right]$. Since

$$
\|F(r, \mu)\|_{H} \leqq\|F(0, \mu)\|_{H}+\sqrt{\beta}\left[{ }_{0}^{\beta}\left\|F^{\prime}(r, \mu)\right\|^{2} d t\right]^{\frac{1}{2}}
$$

for $r \in[0, \beta]$ and $\|F(0, \mu)\|_{H}$ is bounded on $\left[\mu_{1}, \mu_{2}\right],\|F(r, \mu)\|_{H}$ is bounded on $[0, \beta] \times\left[\mu_{1}, \mu_{2}\right]$. This proves the Lemma 4.7. $\quad$ Q. E. D.

To complete the proof of Proposition 4.4, we only need

Lemma 4.8. Let $T(r, \mu)$ be an H-valued function on $[0, \infty) \times \mathbf{R}$ which satisfies the following (a) (d):

(a) $\|T(r, \mu)\|_{H}$ is locally bounded on $[0, \infty) \times \mathbf{R}$.

(b) For any $r \in[0, \infty)$ and $u \in H,(T(r, \mu), u)_{H}$ is locally integrable on $\mathbf{R}$ in the sense of Riemann.

(c) $T(\cdot, \mu) \in \mathbf{D}$ for any $\mu \in \mathbf{R}$. And we have 
(4.52) $\mathscr{L} T(r, \mu)= \begin{cases}\int_{(0, \mu\rfloor} \eta T(r, d \eta) & \text { if } \mu>0, \\ 0 & \text { if } \mu=0, \\ -\int_{(\mu, 0]} \eta T(r, d \eta) & \text { if } \mu<0\end{cases}$

for almost all $r \in[0, \infty)$.

(d) $T(0, \mu)=T^{\prime}(0, \mu)=0$.

Then it follows that $T(r, \mu) \equiv 0$ on $[0, \infty) \times \mathbf{R}$.

Proof. We shall prove the case $\mu>0$. In a similar way we can prove the case $\mu=0$ and the case $\mu<0$. Integrating (4.52) and noting (d), we have for all $u \in H$

$$
\begin{aligned}
& (T(r, \mu), u)_{H} \\
& \quad=\int_{0}^{r} \int_{0}^{t}\left\{((A(s)-\mu) T(s, \mu), u)_{H}+\int_{0}^{\mu}(T(s, \eta), u)_{H} d \eta\right\} d s d t .
\end{aligned}
$$

It follows from (4.53) and (a) that $T(r, \mu) \equiv 0$.

Q.E. D.

Proof of Proposition 4.4. Put $T(r, \mu)=F(r, \mu)-U(r, \mu)$. Then by Lemma 4.6 and Lemma 4.7 we can see that $T(r, \mu)$ satisfies the conditions $(\mathrm{a}) \sim(\mathrm{d})$ in Lemma 4.6. Hence we have $T(r, \mu) \equiv 0$, that is, $F(r, \mu) \equiv U(r, \mu)$.

Now we define an operator-valued function which will play an important role in the eigenfunction expansion of $L_{0}$.

Definition 4.9. Let $I=\left(\mu_{1}, \mu_{2}\right]$ be any finite, half-open interval in R. Then we define a linear operator $\xi(I)$ on $\mathfrak{h}$ into $H$ by

$$
\tilde{\xi}(I) F=P_{1} F^{\prime}(0, I)+B_{2}^{-1} P_{2} F(0, I) .
$$

Proposition 4.10. The operator $\xi(I)$ is a bounded, linear operator taking $\mathfrak{h}$ into $H$ and hence its adjoint $\xi^{*}(I)$ is also a bounded, linear operator taking $H$ into $\mathfrak{h}$.

Let $I_{1}=\left(\mu_{1}, \mu_{2}\right]$ and $I_{2}=\left(\nu_{1}, \nu_{2}\right]$ be finite, half-open intervals in $\mathbf{R}$.

(i) Then the relations 


$$
\left\{\begin{array}{l}
\xi\left(I_{1} \cup I_{2}\right)=\xi\left(I_{1}\right)+\xi\left(I_{2}\right), \\
\xi^{*}\left(I_{1} \cup I_{2}\right)=\xi^{*}\left(I_{1}\right)+\xi^{*}\left(I_{2}\right)
\end{array}\right.
$$

are valid if $I_{1} \cap I_{2}=\phi$.

(ii) We have for arbitrary $I_{1}$ and $I_{2}$

$$
\left\{\begin{array}{l}
E\left(I_{1}\right) \xi^{*}\left(I_{2}\right)=\xi^{*}\left(I_{1} \cap I_{2}\right)=E\left(I_{1} \cap I_{2}\right) \xi^{*}\left(I_{1} \cap I_{2}\right), \\
\xi\left(I_{2}\right) E\left(I_{1}\right)=\xi\left(I_{1} \cap I_{2}\right)=\xi\left(I_{1} \cap I_{2}\right) E\left(I_{1} \cap I_{2}\right) .
\end{array}\right.
$$

Hence $\xi^{*}(I)$ maps $H$ into $\mathscr{D}\left(L_{0}\right)$.

(iii) For arbitrary $I_{1}$ and $I_{2}$ we have

$$
\xi\left(I_{1}\right) \xi^{*}\left(I_{2}\right)=\xi\left(I_{1} \cap I_{2}\right) \xi^{*}\left(I_{1} \cap I_{2}\right) .
$$

Especially if $I_{1} \cap I_{2}=\phi$, we have

$$
\xi\left(I_{1}\right) \xi^{*}\left(I_{2}\right)=0
$$

(iv) For a finite half-open interval $I=\left(\lambda_{1}, \lambda_{2}\right]$ in $\mathbf{R}$, we define an B-valued function $\rho(I)$ by

$$
\rho(I)=\xi(I) \xi^{*}(I) .^{9)}
$$

Then $\rho$ is a symmetric, non-negative definite, finitely additive, operatorvalued interval function.

(v) Put

$$
\xi(\mu)= \begin{cases}\xi((0, \mu]) & \text { if } \mu>0, \\ 0 & \text { if } \mu=0, \quad \rho(\mu)=\xi(\mu) \xi^{*}(\mu) . \\ -\xi((\mu, 0]) & \text { if } \mu<0,\end{cases}
$$

We have $\rho\left(\mu_{2}\right)-\rho\left(\mu_{1}\right)=\rho\left(\left(\mu_{1}, \mu_{2}\right]\right)$ for $\mu_{1}<\mu_{2}$ and $\|\rho(\mu)\|$ is locally bounded on $\mathbf{R}$. Let $U(\mu)$ be an H-valued strongly continuous function. Then for any $\mu \in \mathbf{R} \rho(\mu) U(\mu)$ is at most discontinuous of the first kind in $H$ and right strongly continuous. If $E(\mu)=E(\mu-0)$, then $\rho(\mu) U(\mu)$ is strongly continuous at $\mu$.

Proof. If we can show that $\xi(I)$ is a closed operator, then $\xi(I)$ is 9) Cf. [4], p. 117, (5.22) or [5], p. 931 (2.11). 
seen to be a bounded operator by the closed graph theorem. Let $F_{n}$ be a sequence in $\mathfrak{h}$. Suppose that there exist $F_{0} \in \mathfrak{h}$ and $u_{0} \in H$ such that

$$
\begin{cases}F_{n} \rightarrow F_{0} & \text { in } \mathfrak{h}, \\ \xi(I) F_{n} \rightarrow u_{0} & \text { in } H .\end{cases}
$$

Since $I$ is a finite interval, it follows that

$$
\left\{\begin{array}{l}
E(I) F_{n} \rightarrow E(I) F_{0}, \\
L_{0} E(I) F_{n} \rightarrow L_{0} E(I) F_{0} .
\end{array}\right.
$$

Noting that $L_{0} \leqq L$, we can apply Theorem 3.2 to obtain

$$
\begin{cases}F_{n}(0, I) \rightarrow F_{0}(0, I) & \text { in } H, \\ F^{\prime}{ }_{n}(0, I) \rightarrow F_{0}^{\prime}(0, I) & \text { in } H .\end{cases}
$$

Therefore from the definition of $\xi(I)$ we have

$$
\begin{aligned}
\xi(I) F_{n}= & P_{1} F_{n}^{\prime}(0, I)+B_{2}^{-1} C_{2} P_{2} F_{2}(0, I) \\
& \rightarrow P_{1} F_{0}^{\prime}(0, I)+B_{2}^{-1} C_{2} P_{2} F_{0}(0, I)=\xi(I) F_{0} \quad \text { in } H .
\end{aligned}
$$

Comparison of (4.63) wath (4.60) gives the result that $\xi(I) F_{0}=u_{0}$, i.e. $\xi(I)$ is a closed operator.

Next we shall prove (i) (iv). (i) is obvious by the fact that $E(I)$ is an additive interval function. If $F \in \mathfrak{h}$, then we have

$$
\begin{aligned}
\xi\left(I_{1}\right) E\left(I_{2}\right) F & =P_{1}\left(E\left(I_{1}\right)\left(E\left(I_{2}\right) F\right)\right)^{\prime}(0)+B_{2}^{-1} P_{2}\left(E\left(I_{1}\right)\left(E\left(I_{2}\right) F\right)\right)(0) \\
& =P_{1}\left(E\left(I_{1} \cap I_{2}\right) F\right)^{\prime}(0)+B_{2}^{-1} P_{2}\left(E\left(I_{1} \cap I_{2}\right) F\right)(0) \\
& =\xi\left(I_{1} \cap I_{2}\right) F,
\end{aligned}
$$

which proves the first relation of (4.55). Taking the adjoint in both sides of (4.65), we have

$$
E\left(I_{2}\right) \xi^{*}\left(I_{2}\right)=\xi^{*}\left(I_{1} \cap I_{2}\right),
$$

which proves the second relation of (4.55). Using (ii) we can prove (iii). In fact we have for $u \in H$

$$
\begin{aligned}
\xi\left(I_{1}\right) \xi^{*}\left(I_{2}\right) u & \\
& =P_{1}\left(E\left(I_{1}\right) \xi^{*}\left(I_{2}\right) u\right)^{\prime}(0)+B_{2}^{-1} P_{2}\left(E\left(I_{1}\right) \xi^{*}\left(I_{2}\right) u\right)(0)
\end{aligned}
$$




$$
\begin{aligned}
& =P_{1}\left(E\left(I_{1} \cap I_{2}\right) \xi^{*}\left(I_{1} \cap I_{2}\right) u\right)^{\prime}(0) \\
& \quad+B_{2}^{-1} P_{2}\left(E\left(I_{1} \cap I_{2}\right) \xi^{*}\left(I_{1} \cap I_{2}\right) u\right)(0) \\
& \quad \xi\left(I \cap I_{2}\right) \xi^{*}\left(I_{1} \cap I_{2}\right) u,
\end{aligned}
$$

which proves (iii). Obviously $\rho(I) \geqq 0$ and $\rho(\phi)=0$. If $I_{1} \cap I_{2}=\phi$, then we make use of (4.55) and (4.58) to obtain

$$
\begin{aligned}
\rho\left(I_{1} \cup I_{2}\right) & =\xi\left(I_{1} \cup I_{2}\right) \xi^{*}\left(I_{1} \cup I_{2}\right) \\
& =\left(\xi\left(I_{1}\right)+\xi\left(I_{2}\right)\right)\left(\xi^{*}\left(I_{1}\right)+\xi^{*}\left(I_{2}\right)\right) \\
& =\xi\left(I_{1}\right) \xi^{*}\left(I_{1}\right)+\xi\left(I_{2}\right) \xi^{*}\left(I_{2}\right) \\
& =\rho\left(I_{1}\right)+\rho\left(I_{2}\right) .
\end{aligned}
$$

Thus $\rho$ is finitely additive. Finally we show (v). If $0<\mu<\mu_{0}$ then we have $\rho(\mu) U(\mu)=\xi(\mu) \xi^{*}\left(\left(0, \mu_{0}\right]\right) U(\mu)$. Noting that the strong continuity of $U$, it follows from Lemma 4.5 that $\rho(\mu)$ and $\rho(\mu) U(\mu)$ have the properties stated in ( $v)$ and the proof is complete.

Q.E. D.

In the following proposition, $\xi^{*}(I)$ is represented by $\phi(r, \mu)$ and $\rho(\mu)$.

Proposition 4.11. Let $I=\left(\mu_{1}, \mu_{2}\right]$ be a finite, half-open interval in R. Then the equation

$$
\left(\xi^{*}(I) u\right)(r)=\int_{I} \phi(r, \mu) \rho(d \mu) u
$$

holds for any $u \in H$. The integral exists in the sense of Definition 4.1.

(4.69) is written symbolically in the form

$$
\xi^{*}(I)=\int_{I} \phi(r, \mu) \rho(d \mu)
$$

Proof. Putting $F=\xi^{*}(I) u$ in (4.10) and noting the definition of $\xi(I)$, we have

$$
\left(E(I) \xi^{*}(I) u\right)(r)=\int_{\left(\mu_{1}, \mu_{2}\right]} \phi(r, \mu) \xi(d \mu) \xi^{*}(I) u
$$


It follows from (4.58) that

$$
\left(\xi\left(\eta_{2}\right)-\xi\left(\eta_{1}\right)\right) \xi^{*}(I)=\xi\left(\eta_{2}\right) \xi^{*}\left(\eta_{2}\right)-\xi\left(\eta_{1}\right) \xi^{*}\left(\eta_{1}\right)=\rho\left(\eta_{2}\right)-\rho\left(\eta_{1}\right)
$$

for $\mu_{1} \leqq \eta_{1}<\eta_{2} \leqq \mu_{2}$. And it follows from (4.56) that

$$
E(I) \xi^{*}(I)=\xi^{*}(I) \text {. }
$$

By (4.72) and (4.73) we obtain (4.68) from (4.70).

Q.E. D.

Denote by $C_{0}$ the set of all $H$-valued function $F(r)$ on $[0, \infty)$ such that $F(r)$ has a compact support in $[0, \infty)$ and $F(r)$ is strongly continuous on $[0, \infty)$.

Let us represent $\xi(I)$ by $\phi^{*}(r, \mu)$ and $\rho(\mu)$.

Proposition 4.12. Let $F \in C_{0}$ and $I=\left(\mu_{1}, \mu_{2}\right]$ be a finite half-open interval in $\mathbf{R}$. Then we have

$$
\xi(I) F(r)=\int_{\left(\mu_{1}, \mu_{2}\right]} \rho(d \mu)\left[\int_{0}^{\infty} \phi^{*}(r, \mu) F(r) d r\right]
$$

where for each $r \in[0, \infty)$ and $\mu \in \mathbf{R}, \phi^{*}(r, \mu)$ is the adjoint of $\phi(r, \mu)$.

Proof. Put $U(\mu)=\int_{0}^{\infty} \phi^{*}(r, \mu) F(r) d r$. Since $F \in C_{0}, U(\mu)$ is an $H$ valued smooth function. Hence it follows from (v) of Proposition 4.10 and Lemma 4.2 that the integral $\int_{I} \rho(d \mu) U(\mu)$ exists in the sense of Definition 4.1. Let us prove (4.73). Using (4.69) we obtain for $u \in H$ and $F \in C_{0}$

$$
\begin{aligned}
(\xi(I) F, u)_{H} & =\left(F, \xi^{*}(I) u\right)=\int_{0}^{r_{0}}\left(F(r), \int_{I} \phi(r, \mu) \rho(d \mu) u\right)_{H} d r \\
& =\int_{0}^{r_{0}}\left(\int_{I}(d \mu) \phi^{*}(r, \mu) F(r), u\right)_{H} d r,
\end{aligned}
$$

where we assume the carrier of $F$ is contained in $\left[0, r_{0}\right]$. Making use of the formula of integration by parts (4.4), we obtain from (4.74)

$$
\begin{aligned}
(\xi(I) F, u)_{H}=\int_{0}^{r_{0}}\{ & \left.\left(\rho(\mu) \phi^{*}(r, \mu) F(r), u\right)\right|_{\mu=\mu_{1}} ^{\mu=\mu_{2}} \\
& \left.-\int_{\mu_{1}}^{\mu_{2}}\left(\rho(\mu) \frac{\partial \phi^{*}(r, \mu)}{\partial \mu} F(r), u\right) d \mu\right\} d r
\end{aligned}
$$




$$
\begin{aligned}
& =\left.\left(\rho(\mu) \int_{0}^{r_{0}} \phi^{*}(r, \mu) F(r) d r, u\right)\right|_{\mu=\mu_{1}} ^{\mu=\mu_{2}} \\
& \quad-\int_{\mu_{1}}^{\mu_{2}}\left(\rho(\mu) \int_{0}^{r_{0}} \frac{\partial \phi^{*}(r, \mu)}{\partial \mu} F(r) d r, u\right) d r \\
& =\left(\int_{I} \rho(d \mu)\left[\int_{0}^{r_{0}} \phi^{*}(r, \mu) F(r) d r\right], u\right)_{H},
\end{aligned}
$$

where we used the fact that $\frac{\partial}{\partial \mu} \int_{0}^{r_{0}} \phi^{*}(r, \mu) F(r) d r$ is equal to $\int_{0}^{r_{0}} \frac{\partial \phi^{*}(r, \mu)}{\partial \mu}$ $F(r) d r$ in the strong sense. This proves the proposition.

Q.E. D.

We are now in a position to discuss the eigenfunction expansion for $L_{0}$.

Definition 4.13. Let $U(\mu)$ and $V(\mu)$ be H-valued functions on a finite, closed interval $\left[\mu_{1}, \mu_{2}\right]$ in $\mathbf{R}$. Denote by $\Delta$ a finite subdivision of $\left[\mu_{1}, \mu_{2}\right]$ determined by the points $\eta_{0}, \eta_{1}, \ldots, \eta_{n}$ as in Definition 4.1. $\delta(\Delta)$ means the norm of $\Delta$. Let $\rho(\mu)$ be the operator-valued function defined in (4.68). If the limit

$$
\lim _{\delta(\Delta) \rightarrow 0} \sum_{i=0}^{n-1}\left(\left(\rho\left(\eta_{i+1}\right)-\rho\left(\eta_{i}\right)\right) U\left(\eta_{i}^{\prime}\right), V\left(\eta_{i}^{\prime}\right)\right)_{H}
$$

exists independently of the manner of subdivision and of the choice of the numbers $\eta_{i}^{\prime} \in\left[\eta_{i}, \eta_{i+1}\right]$ then the limit is denoted by

$$
\int_{\left[\mu_{1}, \mu_{2}\right]}(\rho(d \mu) U(\mu), V(\mu))_{H} .
$$

Let us assume that the integral $\int_{\left[\mu, \mu_{2}\right]}(\rho(d \eta) U(\eta), V(\eta))_{H}$ exists for any $\mu \in\left(\mu_{1}, \mu_{2}\right]$ and that the limit $\lim _{\mu \rightarrow \mu_{1}+0} \int_{\left[\mu, \mu_{2}\right]}(\rho(d \eta) U(\eta), V(\eta))_{H}$ exists. Then we put

$$
\int_{\left(\mu_{1}, \mu_{2}\right]}(\rho(d \eta) U(\eta), V(\eta))_{H}=\lim _{\mu \rightarrow \mu_{1}+0} \int_{\left[\mu_{\left., \mu_{2}\right]}\right.}(\rho(d \eta) U(\eta), V(\eta))_{H} .
$$

As for the existence of the integral $\int_{\left[\mu_{1}, \mu_{2}\right]}(\rho(d \eta) U(\eta), V(\eta))_{H}$, we have

Lemma 4.14. Let $U(\mu)$ and $V(\mu)$ be H-valued strongly continuously differentiable functions on $\left[\mu_{1}, \mu_{2}\right]$. Then the integral (4.77) exists and we have 


$$
\begin{gathered}
\int_{\left[\mu_{1}, \mu_{2}\right]}(\rho(d \mu) U(\mu), V(\mu))_{H}=\left(\rho\left(\mu_{2}\right) U\left(\mu_{2}\right), V\left(\mu_{2}\right)\right)_{H} \\
-\left(\rho\left(\mu_{1}\right) U\left(\mu_{1}\right), V\left(\mu_{1}\right)\right)_{H} \\
-\int_{\mu_{1}}^{\mu_{2}}\left\{\left(\rho(\mu) \frac{d U}{d \mu}, V(\mu)\right)_{H}+\left(\rho(\mu) U(\mu), \frac{d V}{d \mu}\right)_{H}\right\} d \mu .
\end{gathered}
$$

Lemma 4.14 will be proved in the Appendix.

Proposition 4.15. Let $F \in C_{0}$ and let $I=\left(\mu_{1}, \mu_{2}\right]$ be a finite halfopened interval in R. Putting

$$
\left(\mathscr{F}_{0} F\right)(\mu)=\int_{0}^{\infty} \phi^{*}(r, \mu) F(r) d r
$$

we have
(i) $(E(I) F)(r)=\int_{I} \phi(r, \mu) \rho(d \mu)\left(\mathscr{F}_{0} F\right)(\mu)$,
(ii) $\|E(I) F\|_{\mathfrak{h}}=\int_{I}\left(\rho(d \mu)\left(\mathscr{F}_{0} F\right)(\mu),\left(\mathscr{F}_{0} F\right)(\mu)\right)_{H}$,

and

$$
\begin{aligned}
&\|F\|_{\mathfrak{h}}^{2}=\lim _{\substack{\mu_{2} \rightarrow \infty \\
\mu_{1} \rightarrow-\infty}} \int_{I}\left(\rho(d \mu)\left(\mathscr{F}_{0} F\right)(\mu),\left(\mathscr{F}_{0} F\right)(\mu)\right)_{H} . \\
& \text { (iii) } \quad L_{0} E(I) F(r)=\int_{I} \mu \phi(r, \mu) \rho(d \mu)\left(\mathscr{F}_{0} F\right)(\mu)
\end{aligned}
$$

for almost all $r \in[0, \infty)$.

Proof. From (v) of Proposition 4.10 and Lemma 4.2 it follows that the integral $\int_{I} \phi(r, \mu) \rho(d \mu)\left(\mathscr{F}_{0} F\right)(\mu)$ exists for every $r \in[0, \infty)$. We shall prove this proposition assuming that $I=\left(0, \mu_{2}\right]$. We can treat the general case in a similar way. Take $u \in H$. By Proposition 4.2 and Proposition 4.12 we have

$$
\begin{aligned}
(E(I) F(r), u)_{H}= & \left(\int_{l} \phi(r, \mu) \xi(d \mu) F, u\right)_{H} \\
= & \left(\phi\left(r, \mu_{2}\right) \xi\left(\mu_{2}\right) F, u\right)-\int_{0}^{\mu_{2}}\left(\frac{\partial \phi(r, \mu)}{\partial \mu} \xi(\mu) F, u\right)_{H} d \mu \\
= & \left(\int_{\left(0, \mu_{2}\right]} \rho(d \mu)\left(\mathscr{F}_{0} F\right)(\mu), \phi^{*}\left(r, \mu_{2}\right) u\right)_{H} \\
& -\int_{0}^{\mu_{2}}\left(\int_{(0, \mu]} \rho(d \eta)\left(\mathscr{F}_{0} F\right)(\eta), \frac{\partial \phi^{*}(r, \mu)}{\partial \mu} u\right)_{H} d \mu .
\end{aligned}
$$


Again we make use of the formula of integration by parts to obtain

$$
\begin{aligned}
& (E(I) F(r), u)_{H}=\left(\rho\left(\mu_{2}\right)\left(\mathscr{F}_{0} F\right)\left(\mu_{2}\right), \phi^{*}\left(r, \mu_{2}\right) u\right)_{H} \\
& -\int_{0}^{\mu_{2}}\left(\rho(\mu) \frac{d\left(\mathscr{F}_{0} F\right)}{d \mu}, \phi^{*}\left(r, \mu_{2}\right) u\right)_{H} \\
& -\int_{0}^{\mu_{2}}\left(\rho(\mu)\left(\mathscr{F}_{0} F\right)(\mu), \frac{\partial \phi^{*}(r, \mu)}{\partial \mu} u\right)_{H} d \mu \\
& +\int_{0}^{\mu_{2}} \int_{0}^{\mu}\left(\rho(\eta) \frac{d\left(\mathscr{F}_{0} F\right)}{d \eta} \frac{\partial \phi^{*}(r, \mu)}{\partial \mu} u\right)_{H} d \eta d \mu \\
& =\left(\phi\left(r, \mu_{2}\right) \rho\left(\mu_{2}\right)\left(\mathscr{F}_{0} F\right)\left(\mu_{2}\right), u\right)_{H} \\
& -\int_{0}^{\mu_{2}}\left(\frac{\partial \phi(r, \mu)}{\partial \mu} \rho(\mu)\left(\mathscr{F}_{0} F\right)(\mu)+\phi(r, \mu) \rho(\mu) \frac{\rho\left(\mathscr{F}_{0} F\right)}{\partial \mu}, u\right)_{H} d \mu \\
& =\left(\int_{(0, \mu]} \phi(r, \mu) \rho(d \mu)\left(\mathscr{F}_{0} F\right)(\mu), u\right)_{H},
\end{aligned}
$$

which implies (i). Let $F \in C_{0}$ and let the carrier of $F$ is contained in $\left[0, r_{0}\right]$. Then from (i) we have

$$
\begin{gathered}
\|E(I) F\|^{2}=(E(I) F, F)=\int_{0}^{r_{0}}\left(\int_{\left(\mu_{1}, \mu_{2}\right]} \phi(r, \mu) \rho(d \mu)\left(\mathscr{F} F_{0}\right)(\mu), F(r)\right) d r \\
=\left.\int_{0}^{r_{0}}\left(\rho(\mu)\left(\mathscr{F}_{0} F\right)(\mu), \phi^{*}(r, \mu) F(r)\right)_{H}\right|_{\mu=\mu^{1}} ^{\mu=\mu_{2}} d r \\
-\int_{0}^{r_{0}} \int_{\mu_{1}}^{\mu_{2}}\left\{\left(\rho(\mu)\left(\mathscr{F}_{0} F\right)(\mu), \frac{\partial \phi^{*}(r, \mu)}{\partial \mu} F(r)\right)_{H}\right. \\
\left.+\left(\rho(\mu) \frac{d\left(\mathscr{F}_{0} F\right)}{d \mu}, \phi^{*}(r, \mu) F(r)\right)_{H}\right\} d \mu d r \\
=\left.\left(\rho(\mu)\left(\mathscr{F}_{0} F\right)(\mu)\left(\mathscr{F}_{0} F\right)(\mu)\right)\right|_{\mu=\mu_{1}} ^{\mu=\mu_{2}} \\
-\int_{\mu_{1}}^{\mu_{2}}\left\{\left(\rho(\mu)\left(\mathscr{F}_{0} F\right)(\mu), \frac{d}{d \mu}\left(\mathscr{F}_{0} F\right)\right)_{H}\right. \\
\left.+\left(\rho(\mu) \frac{d}{d \mu}\left(\mathscr{F}_{0} F\right),\left(\mathscr{F}_{0} F\right)(\mu)\right)_{H}\right\} d \mu \\
=\int_{I}\left(\rho(d \mu)\left(\mathscr{F}_{0}\right) F(\mu),\left(\mathscr{F}_{0} F\right)(\mu)\right)_{H .}
\end{gathered}
$$

Here we have made use of Fubini's theorem on the ordinary Riemann integral and (4.79). It is easy to see that (iii) is obtained from (i). This proves the proposition.

Q.E. D. 
To define the "generalized Fourier transform" associated with $L_{0}$ we must make some preparations.

Denote by $\mathfrak{u}_{0}$ the set of all $H$-valued functions $U(\mu)$ on $\mathbf{R}$ which satisfy the following

(1) $U(\mu)$ has a compact carrier.

(2) $U(\mu)$ is at most discontinuous of the first kind and left continuous.

(3) There exists a finite number of points $\beta_{i}=\beta_{i}(U)(i=1,2, \ldots, N$, $\left.\beta_{1}<\beta_{2}<\cdots<\beta_{N}\right)$ such that if on each closed interval $\left[\beta_{i}, \beta_{i+1}\right](i=1$, $2, \ldots, N-1)$ we define an $H$-valued function

$$
U_{i}(\mu)= \begin{cases}U(\mu) & \text { if } \beta_{i}<\mu \leqq \beta_{i+1}, \\ \underset{\eta \rightarrow \beta_{i}+0}{\mathrm{~s}-\lim _{i} U(\eta)} & \text { if } \mu=\beta_{i},\end{cases}
$$

then $U_{i}(\mu)$ is strongly continuously differentiable on $\left[\beta_{i}, \beta_{i+1}\right](i=1$, $2, \cdots, N-1)$ and $U(\mu)=0$ for $\mu \notin\left[\beta_{1}, \beta_{N}\right]$.

Clearly $\mathfrak{U}_{0}$ is a vector space.

Definition 4.16. Let $U, V \in \mathfrak{U}_{0}$ and let $\beta_{i}(U)(i=1, \ldots, N)$ and $\beta_{j}(V)(j=1,2, \ldots, M)$ be as in (3) of the definition of $\mathfrak{u}_{0}$. We rearrange the points $\beta_{i}(U)$ and $\beta_{j}(V)$ in the order of their magnitude. Thus we obtain a finite number of points $\gamma_{k}(k=1,2, \ldots, K)$ such that $-\infty<\gamma_{1}$ $<\gamma_{2}<\cdots<\gamma_{K}<\infty$ and we have $\left\{\beta_{i}(U)\right\} \cup\left\{\beta_{j}(V)\right\}=\left\{\gamma_{k}\right\}$. Then we define a map $(,)_{\rho}$, which takes $\mathfrak{U}_{0} \times \mathfrak{U}_{0}$ into $\mathbf{C}$ by

$$
(U, V)_{\rho}=\sum_{k=1}^{K-1} \int_{\left(\gamma_{k}, \gamma_{k+1}\right]}(\rho(d \mu) U(\mu), V(\mu))_{H} .
$$

By Lemma $4.14(U, V)_{\rho}$ is well defined.

We can easily show the following relation:

1) $(U+V, W)_{\rho}=(U, W)_{\rho}+(V, W)_{\rho}$,

2) $(\alpha U, V)_{\rho}=(U, \bar{\alpha} V)_{\rho}=\alpha(U, V)_{\rho}(\alpha \in C)$,

3) $(U, V)_{\rho}=\overline{(V, U)_{\rho}}$,

4) $(U, V)_{\rho} \geqq 0$

where $U, V$ and $W$ are elements of $\mathfrak{u}_{0}$. We put $\|U\|_{\rho}=\sqrt{(U, U)_{\rho}}$. Therefore if we denote by $\mathfrak{N}$ the set of all $U \in \mathfrak{U}_{0}$ such that $(U, U)_{\rho}=0$, then the factor space $\mathfrak{U}_{0} / \mathfrak{N}$ becomes a pre-Hilbert space with the inner product 
$(,)_{\rho}$. We define $\mathfrak{h}_{\rho}$ by the completion of $\mathfrak{U}_{0} / \mathfrak{N}$. The inner product and the norm of $\mathfrak{h}_{\rho}$ are also denoted by $(,)_{\rho}$ and \|\|$_{\rho}$ respectively. Then by (ii) of Proposition 4.15 we can see that the operator $\mathscr{F}_{0}$ maps $C_{0}$ into $\mathfrak{h}_{\rho}$ and we have $\left\|\mathscr{F}_{0} F\right\|_{\rho}=\|F\|_{\mathfrak{h}}$ for $F \in C_{0}$. We denote by $\mathscr{F}$ the unique extention to $\mathfrak{h}$ of $\mathscr{F}_{0}$. We call the operator $\mathscr{F}$ the generalized Fourier transform associated with $L_{0}$.

By (ii) of Proposition $4.15 \mathscr{F}$ is an isometric operator on $\mathfrak{h}$.

As for the representation of $\mathscr{F}^{*}$, we have

Proposition 4.17. If $U \in \mathfrak{U}_{0}$, then the relation

$$
\mathscr{F} * U(r)=\sum_{i=1}^{N-1} \int_{\left(\beta_{i} \beta_{\imath+1}\right]} \phi(r, \mu) \rho(d \mu) U(\mu)
$$

holds. Here $\beta_{1}, \beta_{2}, \ldots, \beta_{N}$ are as in (3) of definition of $\mathfrak{U}_{0}$.

Proof. Obviously we can assume that $N=2$. Then for $F \in C_{0}$ we have from Proposition 4.15

$$
\begin{aligned}
\left(F, \mathscr{F}^{*} U\right) & =(\mathscr{F} F, U)_{\rho}=\left(\mathscr{F}_{0} F, U\right)_{\rho} \\
& =\int_{\left.\beta_{1}, \beta_{2}\right]}\left(\rho(d \mu) \int_{0}^{r_{0}} \phi^{*}(r, \mu) F(r) d r, U(\mu)\right)_{H},
\end{aligned}
$$

where we assumed that the carrier of $F$ is contained in $\left[0, r_{0}\right]$. Integrating by parts as in proving Proposition 4.15, we obtain from (4.87)

$$
\left(F, \mathscr{F}^{*} U\right)=\left(F, \int_{\left(\beta_{1}, \beta_{2}\right]} \phi(r, \mu) \rho(d \mu) U(\mu)\right)_{\mathfrak{h}}
$$

for any $F \in C_{0}$, which implies (4.86).

Q. E. D.

We can state an eigenfunction expansion theorem for $L_{0}$.

Theorem 4.18. Let us suppose that a self-adjoint restriction $L_{0}$ of $L$ exists with the resolution of the identity $E(\mu) . \quad$ Let $\mathfrak{h}_{\rho}$ and $\mathscr{F}$ be as in Definition 4.16.

(i) Then $\mathscr{F}$ is a unitary operator from $\mathfrak{h}$ onto $\mathfrak{h}_{\rho}$.

(ii) Let $I$ be an interval in $\mathbf{R}$ and let $\chi_{I}$ be the characteristic function of $I$. Then we have

$$
E(I)=\mathscr{F}^{*} \chi_{1} \mathscr{F}
$$


(iii) $F$ belongs to $\mathscr{D}\left(L_{0}\right)$ if and only if $\mu \cdot(\mathscr{F} F) \in \mathfrak{h}_{\rho}$. If $F \in \mathscr{D}\left(L_{0}\right)$, then we have

$$
L_{0} F=\mathscr{F} * \mu \cdot \mathscr{F} F
$$

Proof. We can easily show (ii) and (iii) using Proposition 4.15. We shall prove (i). We have seen that $\mathscr{F}$ is an isometric operator on $\mathfrak{h}$ into $\mathfrak{h}_{\rho}$. Hence if we show that $\mathscr{F}^{*}$ is isometric, then the proof will be completed. To this end we need

Lemma 4.19. An H-valued function on $\mathbf{R}$ is called a step function if there exists a finite number of mutually disjoint half-open interval $I_{j}=$ $\left(\mu_{j}, \nu_{j}\right]$ and $u_{j} \in H(j=1,2, \ldots, K)$ such that

$$
U(\mu)= \begin{cases}u_{j} & \text { if } \mu \in I_{j}(j=1,2, \ldots, K), \\ 0 & \text { if } u \notin \bigcup_{j=1}^{K} I_{j} .\end{cases}
$$

We denote by $\mathfrak{u}_{s}$ the set of all step functions. Clearly $\mathfrak{U}_{s} \subset \mathfrak{U}_{0}$. Then $\mathfrak{u}_{s}$ is dense in $\mathfrak{h}_{\rho}$.

The proof of Lemma 4.19 will be given in the Appendix.

From Lemma 4.19 we have only to prove that for $U \in \mathfrak{U}_{s}$ we have $\left\|\mathscr{F}^{*} U\right\|_{\mathfrak{\zeta}}=\|U\|_{\rho} \cdot{ }^{10)}$ Let $U(\mu)$ be as in (4.91). Then it follows from Proposition 4.17 and Proposition 4.11 that

$$
\begin{aligned}
\mathscr{F}^{*} U(r) & =\sum_{j=1}^{K} \int_{I_{j}} \phi(r, \mu) \rho(d \mu) U(\mu) \\
& =\sum_{j=1}^{K} \int_{I_{j}} \phi(r, \mu) \rho(d \mu) u_{j} \\
& =\sum_{j=1}^{K} \xi^{*}\left(I_{j}\right) u_{j} .
\end{aligned}
$$

Therefore, noting (4.58), we have

$$
\begin{aligned}
\left\|\mathscr{F}^{*} U\right\|_{\mathfrak{h}}^{2} & =\left(\sum_{j=1}^{K} \xi^{*}\left(I_{j}\right) u_{j} \sum_{j=1}^{K} \xi^{*}\left(I_{j}\right) u_{j}\right)_{\mathfrak{h}} \\
& =\sum_{j, k=1}^{K}\left(\xi\left(I_{k}\right) \xi^{*}\left(I_{j}\right) u_{j}, u_{k}\right)_{H}
\end{aligned}
$$

10) Cf. [4], p. 117. 


$$
\begin{aligned}
& =\sum_{k=1}^{K}\left(\xi\left(I_{k}\right) \xi^{*}\left(I_{k}\right) u_{k}, u_{k}\right)_{H} \\
& =\sum_{k=1}^{K}\left(\rho\left(I_{k}\right) u_{k}, u_{k}\right)_{H} \\
& =\|U\|_{\rho}^{2},
\end{aligned}
$$

which completes the proof.

Q. E. D.

\section{Appendix: The Proofs of Lemma 4.2, 4.3, 4.14 and 4.19}

In this section we shall give the proofs of lemmas which was stated in $\$ 4$. First we shall prove Lemma $4.2,4.3$ and 4.14 which give the sufficient conditions for the existence of integrals defined in Definition 4.1 and 4.13. We shall give the proof of Lemma 4.2 only, because we can prove Lemma 4.3 and Lemma 4.14 in quite a similar way. Next Lemma 4.19 will be proved.

Proof of Lemma 4.2. Let $\Delta$ be a subdivision as in Definition 4.1 and let $\eta_{i}^{\prime} \in\left[\eta_{i}, \eta_{i+1}\right](i=0,1, \ldots, n-1)$. Then we have for $u \in H$

(1) $\quad S(\Delta)=\sum_{i=0}^{n-1}\left(Q\left(\eta_{i}^{\prime}\right)\left(\alpha\left(\eta_{i+1}\right)-\alpha\left(\eta_{i}\right)\right) U\left(\eta_{i}^{\prime}\right), u\right)_{H}$

$$
=\left(Q\left(\eta_{n-1}^{\prime}\right) \alpha\left(\mu_{2}\right) U\left(\eta_{n-1}^{\prime}\right), u\right)_{H}-\left(Q\left(\eta_{0}^{\prime}\right) \alpha\left(\mu_{1}\right) U\left(\eta_{0}^{\prime}\right), u\right)_{H}-\sum_{i=1}^{u-1} I_{i},
$$

where

$$
\begin{aligned}
I_{i}= & \left(Q\left(\eta_{i}^{\prime}\right) \alpha\left(\eta_{i}\right) U\left(\eta_{i}^{\prime}\right)-Q\left(\eta_{i-1}^{\prime}\right) \alpha\left(\eta_{i}\right) U\left(\eta_{i-1}^{\prime}\right), u\right)_{H} \\
= & \left(\left(Q\left(\eta_{i}^{\prime}\right)-Q\left(\eta_{i-1}^{\prime}\right)\right) \alpha\left(\eta_{i}\right) U\left(\eta_{i}^{\prime}\right), u\right)_{H} \\
& \quad+\left(Q\left(\eta_{i-1}^{\prime}\right) \alpha\left(\eta_{i}\right)\left(U\left(\eta_{i}^{\prime}\right)-U\left(\eta_{i-1}^{\prime}\right)\right), u\right)_{H} \\
& \quad I_{i}^{(1)}+I_{i}^{(2)} .
\end{aligned}
$$

Since we have

$$
\begin{aligned}
I_{i}^{(1)} & =\left(\frac{d Q}{d \mu}\left(\eta_{i}\right) \alpha\left(\eta_{i}\right) U\left(\eta_{i}\right), u\right)_{H}\left(\eta_{i}^{\prime}-\eta_{i-1}^{\prime}\right) \\
& +\left(\frac{d Q}{d \mu}\left(\eta_{i}\right) \alpha\left(\eta_{i}\right)\left(U\left(\eta_{i}^{\prime}\right)-U\left(\eta_{i}\right)\right), u\right)_{H}\left(\eta_{i}^{\prime}-\eta_{i-1}^{\prime}\right) \\
& +\int_{\left[\eta_{i-1}^{\prime}, \eta_{i}^{\prime}\right]}\left(\left(\frac{d Q}{d \mu}(\mu)-\frac{d Q}{d \mu}\left(\eta_{i}\right)\right) \alpha\left(\eta_{i}\right) U\left(\eta_{i}^{\prime}\right), u\right)_{H} d \mu,
\end{aligned}
$$


we can see that there exists a positive number $\varepsilon(\Delta)$ such that

$$
\begin{array}{r}
\left|I_{i}^{(1)}-\left(\frac{d Q}{d \mu}\left(\eta_{i}\right) \alpha\left(\eta_{i}\right) U\left(\eta_{i}\right), u\right)_{H}\left(\eta_{i}^{\prime}-\eta_{i-1}^{\prime}\right)\right| \leqq \varepsilon(\Delta)\left(\eta_{i}^{\prime}-\eta_{i-1}^{\prime}\right) \\
(i=1,2, \ldots, n-1),
\end{array}
$$

and

$$
\lim _{\delta(\Delta) \rightarrow 0} \varepsilon(\Delta)=0
$$

Similarly we have

$$
\begin{array}{r}
\left|I_{i}^{(2)}-\left(Q\left(\eta_{i}\right) \alpha\left(\eta_{i}\right) \frac{d U}{d \mu}\left(\eta_{i}\right), u\right)_{H}\left(\eta_{i}^{0}-\eta_{i-1}^{\prime}\right)\right| \leqq \varepsilon(\Delta)\left(\eta_{0}^{\prime}-\eta_{i-1}^{\prime}\right) \\
(i=1,2, \ldots, n-1) .
\end{array}
$$

It follows from (1), (4) and (5) that

$$
\lim _{\delta(\Delta) \rightarrow 0} S(\Delta)=\left(\int_{\left[\mu_{1}, \mu_{2}\right]} Q(\mu) \alpha(d \mu) U(\mu), u\right)_{H}
$$

exists and the formula of integration by parts (4.4) holds for any $u \in H$.

Q. E. D.

Proof of Lemma 4.19. Since $\mathfrak{U}_{0}$ is dense in $\mathfrak{h}_{\rho}$, in order to prove this lemma it is sufficient to show that for an arbitrary $U \in \mathfrak{U}_{0}$ and any $\varepsilon>0$ we can choose $U_{\varepsilon} \in \mathfrak{U}_{s}$ satisfying $\left\|U-U_{\varepsilon}\right\|_{\rho}<\varepsilon$. Further, with no loss of generality, we can assume that there exists a finite, half-open interval $\left(\beta_{1}, \beta_{2}\right]$ such that $U(\mu)=0$ for $\mu \notin\left(\beta_{1} \beta_{2}\right]$ and if we put

$$
U_{1}(\mu)= \begin{cases}U(\mu) & \text { if } \mu \in\left(\beta_{1}, \beta_{2}\right], \\ \underset{\eta \rightarrow \beta_{1}+0}{\mathrm{~s}-\lim _{1}} U(\eta) & \text { if } \mu=\beta_{1},\end{cases}
$$

then $U_{1}(\mu)$ is strongly continuously differentiable on $\left[\begin{array}{ll}\beta_{1} & \beta_{2}\end{array}\right]$. Put

$$
M=\sup _{\beta_{1}<\mu \leqslant \beta_{2}}\left\|\frac{d U}{d \mu}\right\| H
$$

and

$$
L=\sup _{\beta_{1} \leqslant \mu \leqslant \beta_{2}}\|\rho(\mu)\|
$$


Clearly we have $M<\infty$ and by (v) of Proposition 4.10 we have also $L<\infty$. Let us choose a subdivision of $\left[\beta_{1}, \beta_{2}\right]$ by the points $\beta_{1}=\eta_{0}$ $<\eta_{1}<, \ldots,<\eta_{N}=\beta_{2}$ such that

$$
\max _{k=1, \cdots, N}\left(\eta_{k}-\eta_{k-1}\right)<\frac{\varepsilon^{2}}{3\left(\beta_{2}-\beta_{1}\right) L M^{2}}
$$

and let us define $U_{\varepsilon} \in \mathfrak{U}_{s}$ by

$$
U_{\varepsilon}(\mu)= \begin{cases}u_{k} & \text { if } \mu \in\left(\eta_{k}, \eta_{k+1}\right] \quad(k=0,1,2, \ldots, N-1), \\ 0 & \text { if } \mu \notin\left(\beta_{1}, \beta_{2}\right],\end{cases}
$$

where we put $u_{k}=\operatorname{s-lim}_{\eta \rightarrow \eta_{k}+0} U(\eta)(k=0,1,2, \ldots, N-1)$.

It follows from Lemma 4.14 that

$$
\begin{aligned}
\int_{\left(\eta_{k}, \eta_{k+1}\right]} & \left(\rho(d \mu)\left(U(\mu)-U_{\varepsilon}(\mu)\right), U(\mu)-U_{\varepsilon}(\mu)\right)_{H} \\
= & \left.\left(\rho(\mu)\left(U(\mu)-u_{k}\right), U(\mu)-u_{k}\right)\right|_{\mu \eta_{k}+0} ^{\mu=\mu_{k+1}} \\
& -\int_{\eta_{k}}^{\eta_{k+1}}\left(\rho(\mu) \frac{d U}{d \mu}, U(\mu)-u_{k}\right) d \mu \\
& -\int_{\eta_{k}}^{\eta_{k+1}}\left(\rho(\mu)\left(U(\mu)-u_{k}\right), \frac{d U}{d \mu}\right) d \mu \\
= & K_{1}+K_{2}+K_{3} .
\end{aligned}
$$

Since we have

$$
\begin{aligned}
K_{1} & =\left(\rho\left(\eta_{k+1}\right)\left(U\left(\eta_{k+1}\right)-u_{k}\right), U\left(\eta_{k+1}\right)-u_{k}\right)_{H} \\
& =\int_{\eta_{k}}^{\eta_{k+1}} \int_{\eta_{k}}^{\eta_{k+1}}\left(\rho\left(\eta_{k+1}\right) \frac{d U}{d \mu}, \frac{d U}{d \nu}\right)_{H} d \mu d \nu,
\end{aligned}
$$

$K_{1}$ is estimated as follows:

$$
\begin{aligned}
\left|K_{1}\right| & \leqq\left(\eta_{k+1}-\eta_{k}\right)^{2}\left\|\rho\left(_{k+1}\right)\right\|\left(\sup _{\eta_{k}<\mu \leqslant \eta_{k+1}}\left\|\frac{d U}{d \mu}\right\| H\right)^{2} \\
& \leqq\left(\eta_{k+1}-\eta_{k}\right)^{2} L M^{2} \\
& <\left(\eta_{k+1}-\eta_{k}\right) \frac{\varepsilon^{2} L M^{2}}{3\left(\beta_{2}-\beta_{1}\right) L M^{2}}=\frac{\left(\eta_{k+1}-\eta_{k}\right)}{3\left(\beta_{2}-\beta_{1}\right)} \varepsilon^{2}
\end{aligned}
$$


where we used (10). In a similar way we have

$$
\left|K_{2}\right|,\left|K_{3}\right|<\frac{\left(\eta_{k+1}-\eta_{k}\right)}{3\left(\beta_{2}-\beta_{1}\right)} \varepsilon^{2}
$$

From (14) and (15) we obtain

$$
\begin{aligned}
\left\|U-U_{\varepsilon}\right\|^{2} & =\sum_{k=0}^{N-1} \int_{\left(\eta_{k}, \eta_{k+1}\right]}\left(\rho(d \mu)\left(U(\mu)-U_{\varepsilon}(\mu)\right), U(\mu)-U_{\varepsilon}(\mu)\right)_{H} \\
& <\sum_{k=0}^{N-1} \frac{\eta_{k+1}-\eta_{k}}{\beta_{2}-\beta_{1}} \varepsilon^{2}=\varepsilon^{2}
\end{aligned}
$$

which completes the proof.

Q.E. D.

\section{References}

[1] Coddington, E.A. and N. Levinston, Theory of Differential Equations, McGrawHill, New York, 1955.

[2] Gorbačuk, V.I. and M.L. Corbačuk, Expansion in eigenfunctions of a secondorder differential equation with operator coefficient, Dokl. Akad. Nauk SSSR 184, No. 4 (1969), 774-777 (Russian). (Soviet Math. 10, No. 1 (1969), 158-162).

[3] Jäger, W., Ein gewöhnlicher Differentialoperator zweiter Ordnung für Funktionen mit Werten in einem Hilbertraum, Math. Z. 113 (1970), 68-98.

[4] Kodaira, K., On singular solutions of second-order differential operators, I, II, Sugakn 7 (1948) 177-191; Ibid. 2 (1948), 113-139. (Japanese).

[5] The eigenvalue problem for ordinary differential equations of the second order and Heisenberg's theory of S-matrix, Amer. J. Math. 71 (1949), 921 945.

[6] Neumark, M.A., Linear differential operators, Gosudarstr. Izdat. Tehn. -Teo. Lit., Moscow, 1954.

[7] Rofe-Beketov, F.S., Eigenfunction expansions for infinite system of differential equation in non-selfadjoint and self-adjoint cases, Mat. Sb. 51 (93) (1960), 293342 (Russian).

[8] Stone, M.H., Linear transformations in Hilbert spaces and their applicatiosn to analysis, Amer. Math. Soc. Colloquium Pub. 15, New York, 1932.

[9] Titchmarsh, E.C., Eigenfunctions associated with second-order differential equations, part I, Oxford Univ. Press, London, 1946.

[10] Weyl, H., Über gewöhnliche Differentialgleichungen mit Singularitäten und die zugehörigen Entwicklungen willkürlicher Funktionen, Math. Ann. 68 (1910), 220-269.

[11] Yosida, K., On Titchmarsh-Kodaira's formula concerning Weyl-Stone's eigenfunction expansion, Nagoya Math. J. 1, (1950), 49-58. Errata, Ibid. 6 (1953), 187-188. 
